\title{
AN ERROR ANALYSIS METHOD SPP-BEAM AND A CONSTRUCTION GUIDELINE OF NONCONFORMING FINITE ELEMENTS FOR FOURTH ORDER ELLIPTIC PROBLEMS*
}

\author{
Jun $\mathrm{Hu}$ \\ LMAM and School of Mathematical Sciences, Peking University, Beijing 100871, China \\ Email: hujun@math.pku.edu.cn \\ Shangyou Zhang \\ Department of Mathematical Sciences, University of Delaware, Newark, DE 19716, USA \\ Email: szhang@udel.edu
}

\begin{abstract}
Under two hypotheses of nonconforming finite elements of fourth order elliptic problems, we present a side-patchwise projection based error analysis method (SPP-BEAM for short). Such a method is able to avoid both the regularity condition of exact solutions in the classical error analysis method and the complicated bubble function technique in the recent medius error analysis method. In addition, it is universal enough to admit generalizations. Then, we propose a sufficient condition for these hypotheses by imposing a set of in some sense necessary degrees of freedom of the shape function spaces. As an application, we use the theory to design a $P_{3}$ second order triangular $H^{2}$ non-conforming element by enriching two $P_{4}$ bubble functions and, another $P_{4}$ second order triangular $H^{2}$ nonconforming finite element, and a $P_{3}$ second order tetrahedral $H^{2}$ non-conforming element by enriching eight $P_{4}$ bubble functions, adding some more degrees of freedom.

Mathematics subject classification: $65 \mathrm{~N} 30,73 \mathrm{C} 02$.
\end{abstract}

Key words: Nonconforming finite element, A priori error analysis, Biharmonic equation.

\section{Introduction}

We solve a biharmonic equation:

$$
\begin{array}{ll}
\Delta^{2} u=f, & \text { in } \Omega, \\
u=u_{\mathbf{n}}:=\frac{\partial u}{\partial \mathbf{n}}=0, & \text { on } \partial \Omega,
\end{array}
$$

where $\Omega$ is a bounded 2D polygonal domain or 3D polyhedral domain, and $\mathbf{n}$ is the unit outer normal to $\partial \Omega$. Doing integration by parts twice, the weak formulation of (1.1) is: Find $u \in H_{0}^{2}(\Omega)$ such that

$$
a(u, v)=(f, v) \quad \forall v \in H_{0}^{2}(\Omega) .
$$

Here $H_{0}^{2}(\Omega):=\left\{v \in H^{2}(\Omega) \mid v=v_{\mathbf{n}}=0\right.$ on $\left.\partial \Omega\right\}$ and $H^{2}(\Omega)$ is the standard Sobolev space [3]. The bilinear forms are

$$
\begin{aligned}
& a(u, v):=\int_{\Omega} D^{2} u: D^{2} v d \mathbf{x}, \\
& (f, v):=\int_{\Omega} f v d \mathbf{x},
\end{aligned}
$$

\footnotetext{
* Received July 30, 2018 / Revised version received September 13, 2018 / Accepted November 28, 2018 / Published online January 3, 2020 /
} 
where

$$
\begin{aligned}
D^{2} u:= & \left(\begin{array}{ll}
\partial_{x_{1} x_{1}} u & \partial_{x_{1} x_{2}} u \\
\partial_{x_{2} x_{1}} u & \partial_{x_{2} x_{2}} u
\end{array}\right) \text { for } 2 D, \\
D^{2} u:= & \left(\begin{array}{lll}
\partial_{x_{1} x_{1}} u & \partial_{x_{1} x_{2}} u & \partial_{x_{1} x_{3}} u \\
\partial_{x_{2} x_{1}} u & \partial_{x_{2} x_{2}} u & \partial_{x_{2} x_{3}} u \\
\partial_{x_{3} x_{1}} u & \partial_{x_{3} x_{2}} u & \partial_{x_{3} x_{3}} u
\end{array}\right) \text { for } 3 D,
\end{aligned}
$$

with $\partial_{x_{1} x_{2}} u:=\frac{\partial^{2} u}{\partial x_{1} \partial x_{2}}$.

In traditional finite element methods of problem (1.2), degree 2 is the minimum polynomial degree. We need polynomials of degree 2 or above so that the second derivatives are not identically zero in the weak variational form. On a macro-triangle grid, the Powell-Sabin element [14] is a $P_{2} H^{2}$-conforming finite element in 2D. That is, the finite element space is $C_{1}$, a subspace of the $H^{2}$ Sobolev space. The Hsieh-Clough-Tocher $P_{3}$ element is an $H^{2}$-conforming finite element on the 1-to-3 splitting macro-triangle grids, [6]. When the polynomial degree is 5 or above, single-triangle $H^{2}$-conforming elements can be constructed on general triangular grids [2]; when the polynomial degree is 9 or above, single-tetrahedron $H^{2}$-conforming elements can be constructed on general tetrahedral grids [21,22]. On both non-macro-triangle grids and non-macro-tetrahedra grids, Morley element is a $P_{2}$ non-conforming finite element, i.e., the finite element space is not a subspace of the $H^{2}$ space. The remaining gap is the $P_{3}$ and $P_{4}$ non-conforming finite elements for the 4 -th order differential equations in $2 \mathrm{D}$ and the $P_{3}, \cdots$, $P_{8}$ non-conforming finite elements for the 4 -th order differential equations in 3D.

In this paper, we first present two hypotheses of nonconforming finite elements. Then, under them, we generalize the idea of $[10,12]$ to develop a side-patchwise projection based error analysis method (SPP-BEAM for short). Such a method only assumes the basic $H^{2}$ regularity for the exact solution. Compared with the classical a priori error analysis of nonconforming finite elements $[3,6,16]$, the analysis herein applies integration by parts to discrete functions in nonconforming finite element spaces rather than the exact solution of the problem under consideration. This in particular allows to remove the indispensable regularity condition of the exact solution in the classical analysis. Compared with the recent medius analysis of $[8,10,13]$, the analysis herein does not involve the bubble function technique which was first introduced to analyze efficiency of a posteriori error estimators [17]. Note that the bubble function technique will be very complicated for high dimensional cases and high order problems [8].

As an application of the theory, we propose a sufficient condition for these two hypotheses. More precisely, we give a set of in some sense necessary degrees of freedom of the possible shape functions space. Based on these degrees of freedom, we construct a $P_{3}$ second order triangular $H^{2}$ non-conforming finite element by enriching two $P_{4}$ bubble functions and imposing some additional degrees of freedom. As a result, the shape functions space is of 12 dimensions, and the corresponding degrees of freedom are the function value at three vertices and the average of function, the average of the normal derivative, and the first moment of the normal derivative, on three edges. Then, we construct another $P_{4}$, but still of 2 nd order, $H^{2}$ non-conforming element on triangular grids. After that we design a $P_{3}$ second order tetrahedral $H^{2}$ non-conforming finite element by enriching eight $P_{4}$ bubble functions and adding some necessary degrees of freedom. The shape functions space of this three dimensional element is of 28 dimensions, and the corresponding degrees of freedom are the average and the first moment of function on six edges, and the average of function, the average of the normal derivative, and the first moment of the normal derivative, on four faces. We show that the three elements are well-defined 
and converge at the second order. Moreover, we give explicitly the basis functions for them. Numerical tests confirm the theory.

Previously, for 2D, Wang, $\mathrm{Zu}$ and Zhang enriched the $P_{3}$ polynomials by six $P_{6}$ bubble functions in [20], to obtain a 2nd-order triangular $H^{2}$ non-conforming element. Independently, but similarly, Chen and Chen constructed a 2nd-order triangular $H^{2}$ non-conforming element in [4], by enriching the $P_{3}$ polynomials by three $P_{5}$ bubble functions and three $P_{6}$ bubble functions, cf. [4]. Both elements have 16 degrees of freedom on each element. In [7], Gao, Zhang and Wang did construct another 12 dof 2 nd order triangular $H^{2}$ non-conforming element. In their construction, they enriched the $P_{3}$ space by two special $P_{5}$ bubbles, cf. [7], such that the resulting finite element space is $C^{0}$. This construction seems not natural, but intelligent. We numerically compare the two new triangular elements with three existing finite elements of 2 nd order, showing that the new elements are more stable and more accurate. For 3D, see [5] for a second order $P_{3}$ plus $12 P_{8}$ element bubbles $H^{2}$ nonconforming tetrahedron element and [20] for a second order $P_{3}$ plus $12 P_{7}$ element bubbles $H^{2}$ nonconforming tetrahedron element.

\section{Hypothesis and SPP-BEAM}

This section presents hypotheses and consequently the SPP-BEAM of nonconforming finite element methods satisfying the hypotheses. This leads to abstract a priori error estimates. To this end, we introduce some more notation. Let $\mathcal{T}_{h}=\{K\}$ be a regular triangulation on $\Omega$, cf. [3]. Let $\mathcal{E}_{h}$ be the set of $n-1$ dimensional sides of $\mathcal{T}_{h}$, and $\mathcal{E}_{h}(\Omega)$ be the set of internal sides. For each internal $n-1$ dimensional side $e$, let $\omega_{e}$ be the union of two elements sharing the side $e$; for each boundary side $e$, let $\omega_{e}$ be the unique element which takes $e$ as one of its $n-1$ dimensional sides. Given integer $\ell \geq 0$, let $\Pi_{\ell, \omega_{e}} u$ be the $L^{2}$ projection of $u$ to the degree $\ell$ polynomial space on $\omega_{e}$, and $\Pi_{\ell-1, K} u$ be the $L^{2}$ projection of $u$ onto the degree $\ell-1$ polynomial space over element $K$; for the case $\ell=0$, let $\Pi_{\ell-1, K} u=0$.

Let $V_{h, \ell}^{n}$ be a nonconforming finite element space of $H_{0}^{2}(\Omega)$ defined over the mesh $\mathcal{T}_{h}$ with $n=2,3$. The condition of the wellposedness of the discrete problem and a priori error estimate of the finite solution is presented under the following two hypotheses.

Hypothesis 2.1. For any internal $n-1$ dimensional side e, it holds that

$$
\int_{e}\left[\nabla_{h} v\right] \cdot q d s=0 \quad \forall q \in\left(P_{\ell}(e)\right)^{n}, \forall v \in V_{h, \ell}^{n}
$$

here and throughout this paper, $\{\cdot\}$ denotes the average and $[\cdot]$ the jump across a side $e, \nabla_{h}$ and $D_{h}^{2}$ are the discrete counterpart of $\nabla$ and $D^{2}$, respectively, defined piecewise; for any boundary $n-1$ dimensional side $e$, it holds that

$$
\int_{e} \nabla v \cdot q d s=0 \quad \forall q \in\left(P_{\ell}(e)\right)^{n}, \quad \forall v \in V_{h, \ell}^{n}
$$

Hypothesis 2.2. For any internal $n-1$ dimensional side e, it holds that

$$
\int_{e}[v] q d s=0 \quad \forall q \in P_{\ell-1}(e), \quad \forall v \in V_{h, \ell}^{n}
$$

for any boundary $n-1$ dimensional side $e$, it holds that

$$
\int_{e} v q d s=0 \quad \forall q \in P_{\ell-1}(e), \quad \forall v \in V_{h, \ell}^{n} .
$$


For two dimensions, a sufficient condition for both Hypothesis 2.1 and 2.2 is up to additional possible degrees of freedom for the uni-solvency to take the following degrees of freedom on each element $K$ :

1. the moments $\int_{e} \frac{\partial(\cdot)}{\partial \nu_{e}} q d s$ for all $q \in P_{\ell}(e)$ on each edge $e$;

2. the moments $\int_{e}(\cdot) q d s$ for all $q \in P_{\ell-1}(e)$ on each edge $e$;

3. the value of $(\cdot)$ at each vertex.

For three dimensions, a sufficient condition for both Hypothesis 2.1 and 2.2 is up to additional possible degrees of freedom for the uni-solvency to take the following degrees of freedom on each element $K$ :

1. the moments $\int_{e} \frac{\partial(\cdot)}{\partial \nu_{e}} q d s$ for all $q \in P_{\ell}(e)$ on each face $e$;

2. the moments $\int_{e}(\cdot) q d s$ for all $q \in P_{\ell-1}(e)$ on each face $e$;

3. the moments $\int_{s}(\cdot) q d s$ for all $q \in P_{\ell}(s)$ on each edge $s$.

The finite element problem, discretizing the biharmonic equation (1.1), is: Find $u_{h} \in V_{h, \ell}^{n}$ such that

$$
\left(D^{2} u_{h}, D^{2} v_{h}\right)_{h}=\left(f, v_{h}\right) \quad \forall v_{h} \in V_{h, \ell}^{n},
$$

where the discrete inner product is defined element-wise, $(\cdot, \cdot)_{h}=\sum_{K \in \mathcal{T}_{h}}(\cdot, \cdot)_{K}$, and the seminorm $\left|v_{h}\right|_{2, h}=\left(\sum_{K \in \mathcal{T}_{h}}\left(D^{2} v_{h}, D^{2} v_{h}\right)_{K}\right)^{1 / 2}$.

For the analysis, we introduce the projection averaging interpolation operator defined in [16]. To this end, let $V_{h}^{c}$ be the $P_{5}$ Argyris element space for 2D [2] and the $P_{9} H^{2}$ conforming element space for 3D [21,22], respectively. For the $P_{5}$ Argyris element, its degrees of freedom on triangle $K$ are as follows

1. the values of $D^{\ell}(\cdot), \ell=0,1,2$, at each vertex;

2. the first order normal derivative $\frac{\partial(\cdot)}{\partial \mathbf{n}}$ at the mid-point of each edge.

For the $P_{9} H^{2}$ conforming element in 3D, its degrees of freedom on tetrahedron $K$ are as follows

1. the values of $D^{\ell}(\cdot), \ell=0,1,2,3,4$, at each vertex;

2. the first order normal derivatives $\frac{\partial(\cdot)}{\partial \mathbf{n}}$ and $\frac{\partial(\cdot)}{\partial \mathbf{m}}$ at the mid-point of each edge;

3. the second order normal derivatives $\frac{\partial^{2}(\cdot)}{\partial \mathbf{n}^{2}}, \frac{\partial^{2}(\cdot)}{\partial \mathbf{m}^{2}}, \frac{\partial^{2}(\cdot)}{\partial \mathbf{n} \partial \mathbf{m}}$ at 2 equally-distributed internal points of each edge;

4. the value $(\cdot)$ at the mid-point of each face;

5. the first order normal derivatives $\frac{\partial(\cdot)}{\partial \mathbf{n}}$ at three standardly-distributed internal points of each face which are able to uniquely determine a 2D $P_{1}$ polynomial;

6. the values $(\cdot)$ at four standardly-distributed internal points of element which are able to uniquely determine a $3 \mathrm{D} P_{1}$ polynomial. 
where for each edge of $K \mathbf{n}$ and $\mathbf{m}$ are the unit normal vector of the two faces sharing the edge, respectively.

For each degree of freedom $d_{K}$ at point $a$ on the boundary of $K$, let $\omega(a)$ denote the union of elements that share point $a$ and $N(a)$ denote the number of elements in $\omega(a)$. Let $\kappa=2$ for $2 \mathrm{D}$ and $\kappa=4$ for 3D. Then, for any $v \in V_{h, \ell}^{n}$, define the projection average interpolation operator $\tilde{I}_{h}: V_{h, \ell}^{n} \rightarrow V_{h}^{c}$ by: if $a \in \partial \Omega$ and $d_{K}(\phi)=0$ for any $\phi \in C^{\kappa}(\bar{\Omega}) \cap H_{0}^{2}(\Omega)$, then $d_{K}\left(\left.\tilde{I}_{h} v\right|_{K}\right):=0$; otherwise

$$
d_{K}\left(\left.\tilde{I}_{h} v\right|_{K}\right):=\frac{1}{N(a)} \sum_{K^{\prime} \in \omega(a)} d_{K}\left(\left.v\right|_{K^{\prime}}\right)(a) .
$$

Lemma 2.1. Assume that Hypothesis 2.1 and 2.2 hold with $\ell \geq 0$. Then, for $0 \leqslant l \leqslant 2$, it holds that

$$
\left\|D_{h}^{l}\left(v_{h}-\tilde{I}_{h} v_{h}\right)\right\|_{0}^{2} \leq C \sum_{K \in \mathcal{T}_{h}} h_{K}^{2(2-l)}\left\|D^{2} v_{h}\right\|_{0, K}^{2},
$$

for any $v_{h} \in V_{h, \ell}^{n}$. Here and throughout the paper $D_{h}=\nabla_{h}$.

Proof. The proof can be found in [16, Lemma 5.6.4].

Theorem 2.1. Let $u_{h}$ be the solution of (2.1), and $u$ be the solution of (1.1). Assume that Hypothesis 2.1 and 2.2 hold with $\ell \geq 0$, and that the seminorm $\left\|D_{h}^{2} \cdot\right\|_{0}$ defines a norm over the nonconforming finite element space $V_{h, \ell}^{n}$. Then,

$$
\begin{gathered}
\left\|D_{h}^{2}\left(u-u_{h}\right)\right\|_{0} \leq C \inf _{s_{h} \in V_{h, \ell}^{n}}\left\|D_{h}^{2}\left(u-s_{h}\right)\right\|_{0}+C\left(\sum_{e \in \mathcal{E}_{h}}\left\|\left(I-\Pi_{\ell, \omega_{e}}\right) D^{2} u\right\|_{0, \omega_{e}}^{2}\right)^{1 / 2} \\
+C\left(\sum_{K \in \mathcal{T}_{h}} h_{K}^{4}\left\|\left(I-\Pi_{\ell-1, K}\right) f\right\|_{0, K}^{2}\right)^{1 / 2} .
\end{gathered}
$$

Remark 2.1. The SPP-BEAM is closely related to the medius error analysis method in $[8,10$, 13]. The major new idea herein is to introduce the side patch projection operator $\Pi_{\ell, \omega_{e}}$. This enables us to avoid the bubble function technique which is the main tool of $[8,10,13]$. Note that the bubble function technique will become very complicated for high dimensional cases and high order problems.

Proof. Since the seminorm $\left\|D_{h}^{2} \cdot\right\|_{0}$ defines a norm over the nonconforming finite element space $V_{h, \ell}^{n}$, the discrete problem $(2.1)$ is well-defined. For the nonconforming finite element solution, it holds that

$$
\begin{aligned}
& \left(D^{2}\left(s_{h}-u_{h}\right), D^{2}\left(s_{h}-u_{h}\right)\right)_{h} \\
= & \left(D^{2} u, D^{2}\left(s_{h}-u_{h}\right)\right)_{h}-\left(f, s_{h}-u_{h}\right)+\left(D^{2}\left(s_{h}-u\right), D^{2}\left(s_{h}-u_{h}\right)\right)_{h},
\end{aligned}
$$

for any $s_{h} \in V_{h, \ell}^{n}$. By the Schwarz inequality and the triangle inequality,

$$
\begin{aligned}
& \left|s_{h}-u_{h}\right|_{2, h} \leq \sup _{0 \neq v_{h} \in V_{h, \ell}^{n}} \frac{\left(D^{2} u, D^{2} v_{h}\right)_{h}-\left(f, v_{h}\right)}{\left|v_{h}\right|_{2, h}}+\left|u-s_{h}\right|_{2, h}, \\
& \left|u-u_{h}\right|_{2, h} \leq \sup _{0 \neq v_{h} \in V_{h, \ell}^{n}} \frac{\left(D^{2} u, D^{2} v_{h}\right)_{h}-\left(f, v_{h}\right)}{\left|v_{h}\right|_{2, h}}+2\left|u-s_{h}\right|_{2, h} .
\end{aligned}
$$


Let $\tilde{v}_{h}=\tilde{I}_{h} v_{h}$ be the averaging interpolation of $v_{h}$ defined above. Next we shall follow $[8,10,13]$ to use such an averaging interpolation to analyze the first term on the right-hand side of (2.3). This shall allow for an application of the usual Green formulation to a discrete function in the finite element space but not to the exact solution.

$$
\begin{aligned}
& \frac{\left(D^{2} u, D^{2} v_{h}\right)_{h}-\left(f, v_{h}\right)}{\left|v_{h}\right|_{2, h}} \\
= & \frac{\left(D^{2} u, D^{2}\left(v_{h}-\tilde{v}_{h}\right)\right)_{h}-\left(f,\left(v_{h}-\tilde{v}_{h}\right)\right)}{\left|v_{h}\right|_{2, h}} \\
= & \frac{\left(D^{2}\left(u-s_{h}\right), D^{2}\left(v_{h}-\tilde{v}_{h}\right)\right)_{h}+\left(D^{2} s_{h}, D^{2}\left(v_{h}-\tilde{v}_{h}\right)\right)_{h}-\left(f, v_{h}-\tilde{v}_{h}\right)}{\left|v_{h}\right|_{2, h}} \\
\leq & \frac{\left(D^{2} s_{h}, D^{2}\left(v_{h}-\tilde{v}_{h}\right)\right)_{h}-\left(f, v_{h}-\tilde{v}_{h}\right)}{\left|v_{h}\right|_{2, h}}+C\left|u-s_{h}\right|_{2, h},
\end{aligned}
$$

where we use the stability of the averaging interpolation operator $\tilde{I}_{h}$ that

$$
\begin{aligned}
& \left(D^{2}\left(u-s_{h}\right), D^{2}\left(v_{h}-\tilde{v}_{h}\right)\right)_{h} \\
\leq & \left|u-s_{h}\right|_{2, h}\left|v_{h}-\tilde{v}_{h}\right|_{2, h} \leq(1+C)\left|u-s_{h}\right|_{2, h}\left|v_{h}\right|_{2, h} .
\end{aligned}
$$

We start to estimate the consistency error. An integration by parts leads to

$$
\begin{aligned}
& \quad\left(D^{2} s_{h}, D^{2}\left(v_{h}-\tilde{v}_{h}\right)\right)_{h}-\left(f, v_{h}-\tilde{v}_{h}\right) \\
& =\sum_{e \in \mathcal{E}_{h}(\Omega)}\left(\int_{e}\left[D_{h}^{2} s_{h}\right] \mathbf{n} \cdot\left\{\nabla_{h}\left(v_{h}-\tilde{v}_{h}\right)\right\} d s-\int_{e}\left[\operatorname{div}_{h} D_{h}^{2} s_{h}\right] \cdot \mathbf{n}\left\{v_{h}-\tilde{v}_{h}\right\} d s\right) \\
& \quad+\sum_{e \in \mathcal{E}_{h}}\left(\int_{e}\left\{D_{h}^{2} s_{h}\right\} \mathbf{n} \cdot\left[\nabla_{h}\left(v_{h}-\tilde{v}_{h}\right)\right] d s-\int_{e}\left\{\operatorname{div}_{h} D_{h}^{2} s_{h}\right\} \cdot \mathbf{n}\left[v_{h}-\tilde{v}_{h}\right] d s\right) \\
& \quad+\sum_{K \in \mathcal{T}_{h}} \int_{K} \Delta^{2}\left(s_{h}-u\right)\left(v_{h}-\tilde{v}_{h}\right) d \mathbf{x} .
\end{aligned}
$$

Here, $\mathbf{n}$ is a fixed unit normal vector to the side $e$. We estimate the five error terms one by one.

One observation is that $\left[\Pi_{\ell, \omega_{e}} D^{2} u\right]_{e}=0$ for any internal $e$. The main idea here is to use it to analyze the first term on the right-hand side of (2.4) which avoids the bubble function technique used in the medius error analysis from $[8,10,13]$. This leads to

$$
\begin{aligned}
& \int_{e}\left[D_{h}^{2} s_{h}\right] \mathbf{n} \cdot\left\{\nabla_{h}\left(v_{h}-\tilde{v}_{h}\right)\right\} d s \\
= & \int_{e}\left[D_{h}^{2} s_{h}-\Pi_{\ell, \omega_{e}} D^{2} u\right] \mathbf{n} \cdot\left\{\nabla_{h}\left(v_{h}-\tilde{v}_{h}\right)\right\} d s \\
\leq & \left\|\left[D_{h}^{2} s_{h}-\Pi_{\ell, \omega_{e}} D^{2} u\right]\right\|_{0, e}\left\|\left\{\nabla_{h}\left(v_{h}-\tilde{v}_{h}\right)\right\}\right\|_{0, e} \\
\leq & \frac{1}{2}\left\|D^{2} s_{h}-\Pi_{\ell, \omega_{e}} D^{2} u\right\|_{0, e^{+} \cup e^{-}}\left\|\nabla\left(v_{h}-\tilde{v}_{h}\right)\right\|_{0, e^{+} \cup e^{-}} .
\end{aligned}
$$

Here and throughout the paper, $e^{+}$and $e^{-}$denote the restrictions from both side of $e$. Using the inverse inequality, we get

$$
\begin{aligned}
& \left\|D_{h}^{2} s_{h}-\Pi_{\ell, \omega_{e}} D^{2} u\right\|_{0, e^{+} \cup e^{-}} \\
\leq & C\left(h^{-1 / 2}\left\|D_{h}^{2} s_{h}-\Pi_{\ell, \omega_{e}} D^{2} u\right\|_{0, \omega_{e}}+h^{1 / 2}\left|D_{h}^{2} s_{h}-\Pi_{\ell, \omega_{e}} D^{2} u\right|_{1, \omega_{e}}\right) \\
\leq & C h^{-1 / 2}\left(\left\|D_{h}^{2}\left(s_{h}-u\right)\right\|_{0, \omega_{e}}+\left\|\left(I-\Pi_{\ell, \omega_{e}}\right) D^{2} u\right\|_{0, \omega_{e}}\right) .
\end{aligned}
$$


For the other term, we apply the stability of the averaging interpolation operator to get

$$
\begin{aligned}
& \left\|\nabla_{h}\left(v_{h}-\tilde{v}_{h}\right)\right\|_{0, e^{+} \cup e^{-}} \\
\leq & C h^{-1 / 2}\left\|\nabla_{h}\left(v_{h}-\tilde{v}_{h}\right)\right\|_{0, \omega_{e}}+C h^{1 / 2}\left|\nabla_{h}\left(v_{h}-\tilde{v}_{h}\right)\right|_{1, \omega_{e}} \\
\leq & C h^{1 / 2}\left|\nabla_{h}\left(v_{h}-\tilde{v}_{h}\right)\right|_{1, \omega_{e}} \\
\leq & C h^{1 / 2}\left(\left\|D_{h}^{2} v_{h}\right\|_{0, \omega_{e}}+\left\|D_{h}^{2} v_{h}\right\|_{0, \widetilde{\omega}_{e}}\right) \leq C h^{1 / 2}\left\|D_{h}^{2} v_{h}\right\|_{0, \widetilde{\omega}_{e}},
\end{aligned}
$$

where $\widetilde{\omega}_{e}$ is the union of all the elements which shares at least one vertex with the two elements of $\omega_{e}$. Combining the two estimates, we estimate the first term in (2.4) to get

$$
\begin{aligned}
& \sum_{e \in \mathcal{E}_{h}(\Omega)} \int_{e}\left[D_{h}^{2} s_{h}\right] \mathbf{n} \cdot\left\{\nabla_{h}\left(v_{h}-\tilde{v}_{h}\right)\right\} d s \\
\leq & C\left(\sum_{e \in \mathcal{E}_{h}(\Omega)}\left\|\left(I-\Pi_{\ell, \omega_{e}}\right) D^{2} u\right\|_{0, \omega_{e}}^{2}\right)^{1 / 2}\left|v_{h}\right|_{2, h}+C\left\|D_{h}^{2}\left(u-s_{h}\right)\right\|_{0}\left|v_{h}\right|_{2, h} .
\end{aligned}
$$

For the third error term in (2.4), we need to use the $P_{\ell}$ continuity of $\nabla_{h} v_{h}$ in the sense that $\int_{e}\left[\nabla_{h} v_{h}\right] p_{\ell} d s=0$ for any $P_{\ell}$ polynomial $p_{\ell}$.

$$
\begin{aligned}
& \int_{e}\left\{D_{h}^{2} s_{h}\right\} \mathbf{n} \cdot\left[\nabla_{h}\left(v_{h}-\tilde{v}_{h}\right)\right] d s \\
= & \int_{e}\left\{D_{h}^{2} s_{h}-\Pi_{\ell, \omega_{e}} D^{2} u\right\} \mathbf{n} \cdot\left[\nabla_{h}\left(v_{h}-\tilde{v}_{h}\right)\right] d s \\
\leq & \left\|\left\{D_{h}^{2} s_{h}-\Pi_{\ell, \omega_{e}} D^{2} u\right\}\right\|_{0, e}\left\|\left[\nabla_{h}\left(v_{h}-\tilde{v}_{h}\right)\right]\right\|_{0, e} \\
\leq & C h^{-1}\left\|\left\{D_{h}^{2} s_{h}-\Pi_{\ell, \omega_{e}} D^{2} u\right\}\right\|_{0, \omega_{e}}\left\|\left[\nabla_{h}\left(v_{h}-\tilde{v}_{h}\right)\right]\right\|_{0, \omega_{e}} \\
\leq & C\left(\left\|D_{h}^{2}\left(s_{h}-u\right)\right\|_{0, \omega_{e}}+\left\|\left(I-\Pi_{\ell, \omega_{e}}\right) D^{2} u\right\|_{0, \omega_{e}}\right)\left\|D_{h}^{2} v_{h}\right\|_{0, \tilde{\omega}_{e}} .
\end{aligned}
$$

The analysis in the last two steps is the same as that in the last two steps of (2.5). As in (2.6), a summation over all sides gives

$$
\begin{aligned}
& \sum_{e \in \mathcal{E}_{h}} \int_{e}\left\{D_{h}^{2} s_{h}\right\} \mathbf{n} \cdot\left[\nabla_{h}\left(v_{h}-\tilde{v}_{h}\right)\right] d s \\
\leq & C\left(\sum_{e \in \mathcal{E}_{h}}\left\|\left(I-\Pi_{\ell, \omega_{e}}\right) D^{2} u\right\|_{0, \omega_{e}}^{2}\right)^{1 / 2}\left|v_{h}\right|_{2, h}+C\left\|D_{h}^{2}\left(u-s_{h}\right)\right\|_{0}\left|v_{h}\right|_{2, h} .
\end{aligned}
$$

The second error term in (2.4) is estimated similarly to the first term:

$$
\begin{aligned}
& \int_{e}\left[\operatorname{div}_{h} D_{h}^{2} s_{h}\right] \cdot \mathbf{n}\left\{v_{h}-\tilde{v}_{h}\right\} d s \\
= & \int_{e}\left[\operatorname{div}_{h} D_{h}^{2} s_{h}-\operatorname{div} \Pi_{\ell, \omega_{e}} D^{2} u\right] \cdot \mathbf{n}\left\{v_{h}-\tilde{v}_{h}\right\} d s \\
\leq & C h^{-1}\left\|\operatorname{div}_{h} D_{h}^{2} s_{h}-\operatorname{div} \Pi_{\ell, \omega_{e}} D^{2} u\right\|_{0, \omega_{e}}\left\|v_{h}-\tilde{v}_{h}\right\|_{0, \omega_{e}} \\
\leq & C h^{-2}\left\|D_{h}^{2} s_{h}-\Pi_{\ell, \omega_{e}} D^{2} u\right\|_{0, \omega_{e}}\left\|v_{h}-\tilde{v}_{h}\right\|_{0, \omega_{e}} \\
\leq & C\left(\left\|D_{h}^{2}\left(s_{h}-u\right)\right\|_{0, \omega_{e}}+\left\|\left(I-\Pi_{\ell, \omega_{e}}\right) D^{2} u\right\|_{0, \omega_{e}}\right)\left|v_{h}\right|_{2, \tilde{\omega}_{e}} .
\end{aligned}
$$


The fourth error term in (2.4) is analyzed similarly to that of the second term. Using $\int_{e}\left[v_{h}\right] p_{\ell-1} d s=0$ for any $P_{\ell-1}$ polynomial $p_{\ell-1}$, we get

$$
\begin{aligned}
& \int_{e}\left\{\operatorname{div}_{h} D_{h}^{2} s_{h}\right\} \cdot \mathbf{n}\left[v_{h}-\tilde{v}_{h}\right] d s \\
= & \int_{e}\left\{\operatorname{div}_{h} D_{h}^{2} s_{h}-\operatorname{div}_{h} \Pi_{\ell, \omega_{e}} D^{2} u\right\} \cdot \mathbf{n}\left[v_{h}-\tilde{v}_{h}\right] d s \\
\leq & C\left(\left\|D_{h}^{2}\left(s_{h}-u\right)\right\|_{0, \omega_{e}}+\left\|\left(I-\Pi_{\ell, \omega_{e}}\right) D^{2} u\right\|_{0, \omega_{e}}\right)\left|v_{h}\right|_{2, \tilde{\omega}_{e}} .
\end{aligned}
$$

For the fifth error term in (2.4), we have

$$
\int_{K}\left(\Delta^{2} s_{h}-f\right)\left(v_{h}-\tilde{v}_{h}\right) d \mathbf{x} \leq C h_{K}^{2}\left\|\Delta^{2} s_{h}-f\right\|_{0, K}\left|v_{h}\right|_{2, \widetilde{K}}
$$

where $\widetilde{K}$ is the union of elements sharing at least one vertex with $K$. To estimate

$$
\left\|\Delta^{2} s_{h}-f\right\|_{0, K} \leq\left\|\Delta^{2} s_{h}-\Pi_{\ell-1, K} f\right\|_{0, K}+\left\|\left(I-\Pi_{\ell-1, K}\right) f\right\|_{0, K},
$$

we follow the idea of [17] to introduce a $P_{n+1}$ bubble function on element $K$ :

$$
b=\lambda_{1} \cdots \lambda_{n+1},
$$

where $\lambda_{i}$ is a barycentric coordinate on $K$, i.e., the linear function $\lambda_{i}$ assumes value 1 at vertex $\mathbf{x}_{i}$ and 0 on the opposite side. The two norms, the weighted norm and the standard norm, are equivalent.

$$
\begin{aligned}
\left\|\Delta^{2} s_{h}-\Pi_{\ell-1, K} f\right\|_{0, K}^{2} & \leq C\left\|b\left(\Delta^{2} s_{h}-\Pi_{\ell-1, K} f\right)\right\|_{0, K}^{2} \\
& =C\left(\Delta^{2} s_{h}-\Pi_{\ell-1, K} f, w_{K}\right)_{K},
\end{aligned}
$$

where we let

$$
w_{K}=b^{2}\left(\Delta^{2} s_{h}-\Pi_{\ell-1, K} f\right) \in H_{0}^{2}(K) .
$$

In order to use the inverse estimate, we need the following decomposition:

$$
\left(\Delta^{2} s_{h}-\Pi_{\ell-1, K} f, w_{K}\right)_{K}=\left(\Delta^{2} s_{h}-f, w_{K}\right)_{K}+\left(\left(I-\Pi_{\ell-1, K}\right) f, w_{K}\right)_{K} .
$$

We apply the integration by parts twice on one element to get

$$
\begin{aligned}
\left(\Delta^{2} s_{h}-f, w_{K}\right) & =\left(D^{2} s_{h}, D^{2} w_{K}\right)-\left(D^{2} u, D^{2} w_{K}\right) \\
& \leq\left|u-s_{h}\right|_{2, K}\left|w_{K}\right|_{2, K} .
\end{aligned}
$$

Combining the above two inequalities, we obtain

$$
\left(\Delta^{2} s_{h}-\Pi_{\ell-1, K} f, w_{K}\right) \leq\left(C h_{K}^{-2}\left|u-s_{h}\right|_{2, K}+\left\|\left(I-\Pi_{\ell-1, K}\right) f\right\|_{0, K}\right)\left|w_{K}\right|_{0, K} .
$$

By (2.9)-(2.11), we have

$$
\left\|\Delta^{2} s_{h}-f\right\|_{0, K} \leq C h_{K}^{-2}\left|u-s_{h}\right|_{2, K}+C\left\|\left(I-\Pi_{\ell-1, K}\right) f\right\|_{0, K} .
$$

By (2.8), the fifth term of (2.4) is bounded by

$$
\begin{aligned}
& \sum_{K \in \mathcal{T}_{h}} \int_{K} \Delta^{2}\left(s_{h}-u\right)\left(v_{h}-\tilde{v}_{h}\right) d \mathbf{x} \\
\leq & C\left(\left\|D_{h}^{2}\left(u-s_{h}\right)\right\|_{0}+\left(\sum_{K \in \mathcal{T}_{h}} h_{K}^{4}\left\|\left(I-\Pi_{\ell-1, K}\right) f\right\|_{0, K}^{2}\right)^{1 / 2}\right)\left|v_{h}\right|_{2, h} .
\end{aligned}
$$

Putting together the above five bounds into (2.4), we obtain the estimate of the finite element solution in the theorem. 


\section{A $P_{3}+2 B_{4}$ Element in $2 \mathrm{D}$}

On each triangle, the finite element shape functions are formed by the following polynomials

$$
P_{3}^{+}(K):=\operatorname{span}\left\{P_{3}\left(x_{1}, x_{2}\right), x_{1} P_{3,0}(K), x_{2} P_{3,0}(K)\right\}
$$

where $P_{3,0}(K)$ denotes the space of $P_{3}$ polynomials vanishing on $\partial K$. Note that $P_{3,0}(K):=$ $\operatorname{span}\left\{\lambda_{1} \lambda_{2} \lambda_{3}\right\}$ where $\lambda_{i}$ is the barycentric coordinate for vertex $\mathbf{x}_{i}$, a linear polynomial vanishing on one edge. The dual basis of the finite element space, i.e., the degrees of freedom of the finite element, is

$$
\begin{aligned}
& v\left(\mathbf{x}_{i}\right) \quad \text { at three vertices of } K, \\
& \int_{e_{j}} v d s, \int_{e_{j}} \partial_{\mathbf{n}} v d s, \int_{e_{j}} s \partial_{\mathbf{n}} v d s \quad \text { on three edges of } K .
\end{aligned}
$$

The global finite element space is defined by

$$
\begin{aligned}
& V_{h, H Z 12}:=\left\{v \in L^{2}(\Omega)|v|_{K} \in P_{3}^{+}(K), \quad v \text { is continuous at internal vertices of } \mathcal{T}_{h},\right. \\
& \int_{e} v d s, \int_{e} \partial_{\mathbf{n}} v d s \text { and } \int_{e} s \partial_{\mathbf{n}} v d s \text { are continuous across internal edges of } \mathcal{T}_{h}, \\
& v\left(\mathbf{x}_{i}\right)=\int_{e} v d s=\int_{e} \partial_{\mathbf{n}} v d s=\int_{e} s \partial_{\mathbf{n}} v d s=0 \\
& \text { at boundary vertices and edges of } \left.\mathcal{T}_{h}\right\} \text {. }
\end{aligned}
$$

Theorem 3.1. The 12 degrees of freedom in (3.1a) uniquely determine a polynomial of $P_{3}^{+}(K)$, defined in (3.2).

Proof. Let $u_{h} \in P_{3}^{+}(K)$ such that the 12 degrees of freedom of $u_{h}$ in (3.1a) and (3.1b) having zero value. We need to show $u_{h}=0$. On a general triangle $K=\Delta \mathbf{x}_{1} \mathbf{x}_{2} \mathbf{x}_{3}$, we recall that $\lambda_{i}$ is the barycentric coordinate, that is a linear function having values

$$
\lambda_{i}\left(\mathbf{x}_{j}\right)=\delta_{i j}, \quad i, j=1,2,3 .
$$

Then we can choose such a homogeneous basis for the space of the $P_{3}+2 B_{4}$ element that

$$
u_{h}=\sum_{i=1}^{12} u_{i} b_{i}(\mathbf{x})
$$

where

$$
\begin{array}{lll}
b_{1}=\lambda_{1}^{3}, & b_{2}=\lambda_{2}^{3}, & b_{3}=\lambda_{3}^{3}, \\
b_{4}=\lambda_{1}^{2} \lambda_{2}, & b_{5}=\lambda_{1} \lambda_{2}^{2}, & b_{6}=\lambda_{2}^{2} \lambda_{3}, \\
b_{7}=\lambda_{2} \lambda_{3}^{2}, & b_{8}=\lambda_{3}^{2} \lambda_{1}, & b_{9}=\lambda_{3} \lambda_{1}^{2}, \\
b_{10}=\lambda_{1}^{2} \lambda_{2} \lambda_{3}, & b_{11}=\lambda_{1} \lambda_{2}^{2} \lambda_{3}, & b_{12}=\lambda_{1} \lambda_{2} \lambda_{3}^{2} .
\end{array}
$$

At $\mathbf{x}_{1}$, all $b_{i}\left(\mathbf{x}_{1}\right)=0$ except $i=1$. So the three vertex values would force $u_{1}=u_{2}=u_{3}=0$ in (3.3). Next, when restricted on an edge $e_{1}$, only two basis functions $b_{6}$ and $b_{7}$ are nonzero. 


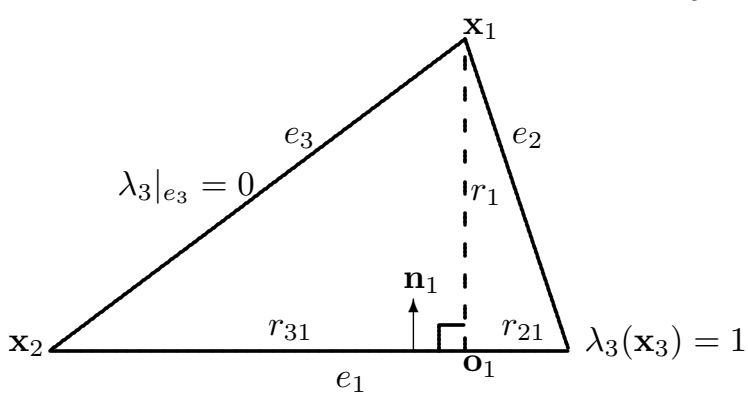

Fig. 3.1. Computing the normal derivative $\partial_{1} \lambda_{3}=\nabla \lambda_{3} \cdot \mathbf{n}_{1}$.

By computing the zeroth moment on $e_{1}$, we get

$$
\int_{e_{1}} b_{7} d s=\int_{e_{1}} b_{6} d s=\frac{1}{12}\left|e_{1}\right|
$$

A combination of two basis leads to $\int_{e_{1}}\left(b_{6}-b_{7}\right) d s=0$. So we change a basis for the expansion of $u_{h}$ in (3.3) to

$$
u_{h}=\tilde{c}_{4} \tilde{b}_{4}+\tilde{c}_{6} \tilde{b}_{6}+\tilde{c}_{8} \tilde{b}_{8}+\tilde{c}_{10} \tilde{b}_{10}+\tilde{c}_{11} \tilde{b}_{11}+\tilde{c}_{12} \tilde{b}_{12}
$$

where

$$
\begin{aligned}
& \tilde{b}_{4}=\left(\lambda_{1}^{2} \lambda_{2}-\lambda_{1} \lambda_{2}^{2}\right), \quad \tilde{b}_{6}=\left(\lambda_{2}^{2} \lambda_{3}-\lambda_{2} \lambda_{3}^{2}\right), \quad \tilde{b}_{8}=\left(\lambda_{3}^{2} \lambda_{1}-\lambda_{3} \lambda_{1}^{2}\right), \\
& \tilde{b}_{10}=\lambda_{1}^{2} \lambda_{2} \lambda_{3}, \quad \tilde{b}_{11}=\lambda_{1} \lambda_{2}^{2} \lambda_{3}, \quad \tilde{b}_{12}=\lambda_{1} \lambda_{2} \lambda_{3}^{2} .
\end{aligned}
$$

Next, consider the 0th and 1st moments of $\partial_{1} u_{h}=\nabla u_{h} \cdot \mathbf{n}_{1}$ on an edge $e_{1}$. For the linear functions $\lambda_{1}$ and $\lambda_{3}$, cf. Fig. 3.1,

$$
\begin{aligned}
& \partial_{1} \lambda_{1}=\nabla \lambda_{1} \cdot \mathbf{n}_{1}=\frac{1}{r_{1}}, \\
& \partial_{1} \lambda_{3}=-\frac{r_{31}}{r_{1}\left|e_{1}\right|}, \quad \partial_{1} \lambda_{2}=-\frac{\left|e_{1}\right|-r_{31}}{r_{1}\left|e_{1}\right|} .
\end{aligned}
$$

Consequently, we have

$$
\begin{aligned}
& \int_{e_{1}} \partial_{1} \tilde{b}_{6} s d s=\int_{e_{1}}\left[-\frac{\left|e_{1}\right|-r_{31}}{r_{1}\left|e_{1}\right|}\left(2 \lambda_{2} \lambda_{3}-\lambda_{3}^{2}\right)-\frac{r_{31}}{r_{1}\left|e_{1}\right|}\left(\lambda_{2}^{2}-2 \lambda_{2} \lambda_{3}\right)\right] s d s \\
= & \frac{\left|e_{1}\right|^{2}}{r_{1}} \int_{0}^{1}\left[-\left(1-\tilde{r}_{31}\right)\left(2(1-t) t-t^{2}\right)-\tilde{r}_{31}\left((1-t)^{2}-2(1-t) t\right)\right] t d t \\
= & \frac{\left|e_{1}\right|^{2}}{r_{1}} \frac{1}{12},
\end{aligned}
$$

where $\tilde{r}_{31}:=r_{31} /\left|e_{1}\right|:=\left|\mathbf{x}_{2} \mathbf{o}_{1}\right| /\left|e_{1}\right|$. Note that the answer is independent of $r_{31}$. Similarly, we get

$$
\int_{e_{1}} \partial_{1}\left(\begin{array}{c}
\tilde{b}_{6} \\
\tilde{b}_{8} \\
\tilde{b}_{4} \\
\tilde{b}_{11} \\
\tilde{b}_{12}
\end{array}\right)\left(\begin{array}{ll}
s & \hat{s}
\end{array}\right) d s=\frac{\left|e_{1}\right|^{2}}{60 r_{1}}\left(\begin{array}{cc}
5 & -5 \\
15 & 5 \\
-5 & -15 \\
2 & 3 \\
3 & 2
\end{array}\right),
$$


where $\hat{s}=\left|e_{1}\right|-s$. The two columns of the matrix make two equations for the unknown $\tilde{c}_{i}$. Rotating the above calculation, on $e_{2}$ and $e_{3}$, together we would get 6 equations that

$$
\left(\begin{array}{ccc|ccc}
-5 & 5 & 15 & & 2 & 3 \\
-15 & -5 & 5 & & 3 & 2 \\
15 & -5 & 5 & 3 & & 2 \\
5 & -15 & -5 & 2 & & 3 \\
5 & 15 & -5 & 2 & 3 & \\
-5 & 5 & -15 & 3 & 2
\end{array}\right)\left(\begin{array}{c}
\tilde{c}_{4} \\
\tilde{c}_{6} \\
\tilde{c}_{8} \\
\tilde{c}_{10} \\
\tilde{c}_{11} \\
\tilde{c}_{12}
\end{array}\right)=0
$$

The determinant of the coefficient matrix is -135000 . Thus we have a unique solution $\tilde{c}_{i}=0$ and $u_{h}=0$.

Theorem 3.2. With $V_{h, \ell}^{2}=V_{h, H Z 12}$ in (2.1), the finite element equations have a unique solution. Moreover,

$$
\left\|D_{h}^{2}\left(u-u_{h}\right)\right\|_{0} \leq C h^{2}|u|_{4},
$$

provided that $u \in H^{4}(\Omega) \cap H_{0}^{2}(\Omega)$.

Proof. For the finite square system of equations, the uniqueness implies the existence of a solution. We show a unique solution of (2.1) when $f=0$. Letting $v_{h}=u_{h}$ in (2.1), we get

$$
\sum_{K \in \mathcal{T}_{h}}\left|D^{2} u_{h}\right|_{0, K}^{2}=0
$$

As all second derivatives of $u_{h}$ are zero, $u_{h}$ is a linear function on each triangle $K$. Since $u_{h}$ is continuous at the vertices of $\mathcal{T}_{h}, u_{h}$ is a function in the space $H_{0}^{1}(\Omega)$. As $\nabla u_{h}$ is continuous at the midpoints of the internal edges and vanishes at the midpoints of the boundary edges, $\nabla u_{h}=0$ and consequently $u_{h}=0$. The error estimate follows from the abstract theory in Theorem 2.1.

\section{The $P_{4}$ Finite Element in $2 \mathrm{D}$}

On a triangular grid, the dual basis of the $P_{4}$ finite element space, i.e., the degrees of freedom of the finite element, is

$$
\begin{array}{ll}
v\left(\mathbf{x}_{i}\right) \quad \text { at three vertices of } K, & \\
\int_{e_{j}} v d s, \quad \int_{e_{j}} \partial_{\mathbf{n}} v d s, \quad \int_{e_{j}} s \partial_{\mathbf{n}} v d s & \text { on three edges of } K, \\
\int_{K} v d x, \quad \int_{K} x_{1} v d x, \quad \int_{K} x_{2} v d x & \text { on the triangle } K .
\end{array}
$$

The global finite element space is defined by

$$
\begin{aligned}
& V_{h, H Z 15}:=\left\{v \in L^{2}(\Omega)|v|_{K} \in P_{4}(K), \quad v \text { is continuous at internal vertices of } \mathcal{T}_{h},\right. \\
& \int_{e} v d s, \int_{e} \partial_{\mathbf{n}} v d s \text { and } \int_{e} s \partial_{\mathbf{n}} v d s \quad \text { are continuous across internal edges of } \mathcal{T}_{h}, \\
& v\left(\mathbf{x}_{i}\right)=\int_{e} v d s=\int_{e} \partial_{\mathbf{n}} v d s=\int_{e} s \partial_{\mathbf{n}} v d s=0 \\
&\text { at boundary vertices and edges of } \left.\mathcal{T}_{h}\right\} .
\end{aligned}
$$


Theorem 4.1. The 15 degrees of freedom in (4.1) uniquely determine a $P_{4}$ polynomial in the space $V_{h, H Z 15}$ of $(4.2)$.

Proof. Let $u_{h} \in P_{4}$ such that the 15 degrees of freedom of $u_{h}$ in (4.1) having zero value. We need to show $u_{h}=0$. On a general triangle $K=\triangle \mathbf{x}_{1} \mathbf{x}_{2} \mathbf{x}_{3}$, we can choose a homogeneous basis for the space of $P_{4}$ such that

$$
u_{h}=\sum_{i=1}^{15} u_{i} b_{i}(\mathbf{x})
$$

where

$$
\begin{array}{lll}
b_{1}=\lambda_{1}^{4}, & b_{2}=\lambda_{2}^{4}, & b_{3}=\lambda_{3}^{4}, \\
b_{4}=\lambda_{1}^{3} \lambda_{2}, & b_{5}=\lambda_{1}^{2} \lambda_{2}^{2}, & b_{6}=\lambda_{1} \lambda_{2}^{3}, \\
b_{7}=\lambda_{2}^{3} \lambda_{3}, & b_{8}=\lambda_{2}^{2} \lambda_{3}^{2}, & b_{9}=\lambda_{2} \lambda_{3}^{3}, \\
b_{10}=\lambda_{3}^{3} \lambda_{1}, & b_{11}=\lambda_{3}^{2} \lambda_{1}^{2}, & b_{12}=\lambda_{3} \lambda_{1}^{3}, \\
b_{13}=\lambda_{1}^{2} \lambda_{2} \lambda_{3}, & b_{14}=\lambda_{1} \lambda_{2}^{2} \lambda_{3}, & b_{15}=\lambda_{1} \lambda_{2} \lambda_{3}^{2} .
\end{array}
$$

At $\mathbf{x}_{1}$, all $b_{i}\left(\mathbf{x}_{1}\right)=0$ except $i=1$. So the three vertex values would force $u_{1}=u_{2}=u_{3}=0$ in (4.3). Next, when restricted on an edge $e_{1}$, only three basis functions $b_{7}, b_{8}$, and $b_{9}$ are nonzero. By computing the zeroth moment on $e_{1}$, we get

$$
\int_{e_{1}} b_{7} d s=\int_{e_{1}} b_{9} d s=\frac{\left|e_{1}\right|}{20}, \quad \int_{e_{1}} b_{8} d s=\frac{\left|e_{1}\right|}{30}
$$

A combination of two basis yields $\int_{e_{1}}\left(2 b_{7}-3 b_{8}\right) d s=0$. The constraint of zero 0th moment on $e_{1}$ would force the coefficient of the only non-zero term $b_{8}$ to be zero. So we change a basis for the expansion of $u_{h}$ in (4.3) to

$$
u_{h}=\tilde{c}_{4} \tilde{b}_{4}+\tilde{c}_{6} \tilde{b}_{6}+\tilde{c}_{7} \tilde{b}_{7}+\tilde{c}_{9} \tilde{b}_{9}+\tilde{c}_{10} \tilde{b}_{10}+\tilde{c}_{12} \tilde{b}_{12}+\tilde{c}_{13} \tilde{b}_{13}+\tilde{c}_{14} \tilde{b}_{14}+\tilde{c}_{15} \tilde{b}_{15}
$$

where

$$
\begin{array}{ll}
\tilde{b}_{4}=\left(2 \lambda_{1}^{3} \lambda_{2}-3 \lambda_{1}^{2} \lambda_{2}^{2}\right), & \tilde{b}_{6}=\left(2 \lambda_{1} \lambda_{2}^{3}-3 \lambda_{1}^{2} \lambda_{2}^{2}\right), \\
\tilde{b}_{7}=\left(2 \lambda_{2}^{3} \lambda_{3}-3 \lambda_{2}^{2} \lambda_{3}^{2}\right), & \tilde{b}_{9}=\left(2 \lambda_{2} \lambda_{3}^{3}-3 \lambda_{2}^{2} \lambda_{3}^{2}\right), \\
\tilde{b}_{10}=\left(2 \lambda_{3}^{3} \lambda_{1}-3 \lambda_{3}^{2} \lambda_{1}^{2}\right), & \tilde{b}_{12}=\left(2 \lambda_{3} \lambda_{1}^{3}-3 \lambda_{3}^{2} \lambda_{1}^{2}\right), \\
\tilde{b}_{13}=\lambda_{1}^{2} \lambda_{2} \lambda_{3}, \quad \tilde{b}_{14}=\lambda_{1} \lambda_{2}^{2} \lambda_{3}, \quad \tilde{b}_{15}=\lambda_{1} \lambda_{2} \lambda_{3}^{2} .
\end{array}
$$

Next, consider the 0 th and 1 st moments of $\partial_{1} u_{h}=\nabla u_{h} \cdot \mathbf{n}_{1}$ on an edge $e_{1}$. For the linear functions $\lambda_{1}$ and $\lambda_{3}$, cf. Figure 3.1,

$$
\partial_{1} \lambda_{1}=\nabla \lambda_{1} \cdot \mathbf{n}_{1}=\frac{1}{r_{1}}, \quad \partial_{1} \lambda_{3}=-\frac{r_{31}}{r_{1}\left|e_{1}\right|}, \quad \partial_{1} \lambda_{2}=-\frac{\left|e_{1}\right|-r_{31}}{r_{1}\left|e_{1}\right|} .
$$


Consequently, we have

$$
\begin{aligned}
\int_{e_{1}} \partial_{1} \tilde{b}_{7} s d s= & \int_{e_{1}}\left[-\frac{\left|e_{1}\right|-r_{31}}{r_{1}\left|e_{1}\right|}\left(6 \lambda_{2}^{2} \lambda_{3}-6 \lambda_{2} \lambda_{3}^{2}\right)-\frac{r_{31}}{r_{1}\left|e_{1}\right|}\left(2 \lambda_{2}^{3}-6 \lambda_{2}^{2} \lambda_{3}\right)\right] s d s \\
= & \frac{\left|e_{1}\right|^{2}}{r_{1}} \int_{0}^{1}\left[-\left(1-\tilde{r}_{31}\right)\left(6(1-t)^{2} t-6(1-t) t^{2}\right)\right. \\
& \left.\quad-\tilde{r}_{31}\left(2(1-t)^{3}-6(1-t)^{2} t\right)\right] t d t \\
= & \frac{\left|e_{1}\right|^{2}}{r_{1}} \frac{1}{10},
\end{aligned}
$$

where $\tilde{r}_{31}=r_{31} /\left|e_{1}\right|$. Note that the answer is independent of $r_{31}$. Similarly, we get

$$
\int_{e_{1}} \partial_{1}\left(\begin{array}{c}
\tilde{b}_{7} \\
\tilde{b}_{9} \\
\tilde{b}_{10} \\
\tilde{b}_{6} \\
\tilde{b}_{15} \\
\tilde{b}_{14}
\end{array}\right)\left(\begin{array}{ll}
s & \hat{s}
\end{array}\right) d s=\frac{\left|e_{1}\right|^{2}}{r_{1}}\left(\begin{array}{cc}
\frac{1}{10} & -\frac{1}{10} \\
-\frac{1}{10} & \frac{1}{10} \\
\frac{4}{10} & \frac{1}{10} \\
\frac{1}{10} & \frac{4}{10} \\
\frac{1}{20} & \frac{1}{30} \\
\frac{1}{30} & \frac{1}{20}
\end{array}\right)
$$

where $\hat{s}=\left|e_{1}\right|-s$. The two columns of the matrix make two equations for the unknown $\tilde{c}_{i}$. Rotating the above calculation, on $e_{2}$ and $e_{3}$, we would get 6 equations that

$$
\left(\begin{array}{cccccc:ccc} 
& 6 & 6 & -6 & 24 & & & 2 & 3 \\
& 24 & -6 & 6 & 6 & & & 3 & 2 \\
24 & & & 6 & 6 & -6 & 3 & & 2 \\
6 & & & 24 & -6 & 6 & 2 & & 3 \\
6 & -6 & 24 & & & 6 & 2 & 3 & \\
-6 & 6 & 6 & & & 24 & 3 & 2
\end{array}\right)\left(\begin{array}{c}
\tilde{c}_{4} \\
\tilde{c}_{6} \\
\tilde{c}_{7} \\
\tilde{c}_{9} \\
\tilde{c}_{10} \\
\tilde{c}_{12} \\
\tilde{c}_{13} \\
\tilde{c}_{14} \\
\tilde{c}_{15}
\end{array}\right)=0
$$

The reduced row-echelon form is

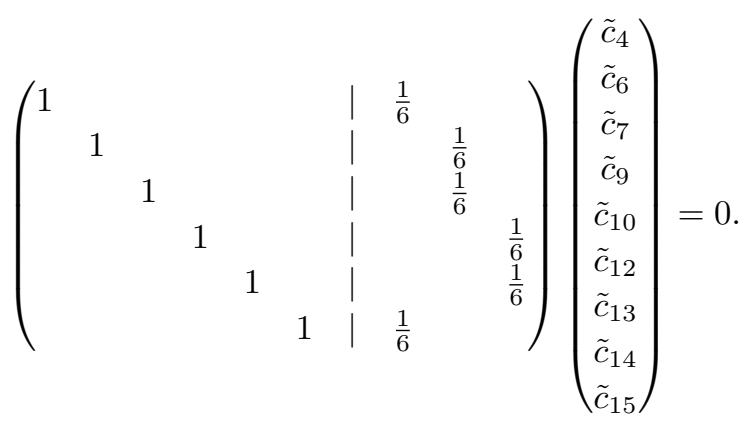

Thus, if we choose basis functions $\hat{b}_{i}=\tilde{b}_{i}, i \leq 12$, and

$$
\begin{aligned}
\hat{b}_{13} & =\tilde{b}_{13}-\frac{1}{6} \tilde{b}_{4}-\frac{1}{6} \tilde{b}_{12}, \quad \hat{b}_{14}=\tilde{b}_{14}-\frac{1}{6} \tilde{b}_{6}-\frac{1}{6} \tilde{b}_{7}, \\
\hat{b}_{15} & =\tilde{b}_{15}-\frac{1}{6} \tilde{b}_{8}-\frac{1}{6} \tilde{b}_{10},
\end{aligned}
$$


then the expansion of $u_{h}$ is forced to a simple form, by above 12 conditions, that

$$
u_{h}=\hat{c}_{13} \hat{b}_{13}+\hat{c}_{14} \hat{b}_{14}+\hat{c}_{15} \hat{b}_{15}
$$

Finally, evaluating $u_{h}$ at the three internal points, $\left(2 \mathbf{x}_{i}+\mathbf{x}_{j}+\mathbf{x}_{k}\right) / 4$, we get

$$
\frac{1}{1536}\left(\begin{array}{lll}
16 & 21 & 21 \\
21 & 16 & 21 \\
21 & 21 & 16
\end{array}\right)\left(\begin{array}{l}
\hat{c}_{13} \\
\hat{c}_{14} \\
\hat{c}_{15}
\end{array}\right)=0 .
$$

Adding 5 to the diagonal entries of the above matrix, the eigenvalues are $0,0,3 \times 21$. So the eigenvalues of the above matrix are $-5,-5,58$, non zero. Thus, $\hat{c}_{i}=0$ and $u_{h}=0$.

Theorem 4.2. With $V_{h, \ell}^{2}=V_{h, H Z 15}$ in (2.1), the finite element equations have a unique solution. Moreover,

$$
\left\|D_{h}^{2}\left(u-u_{h}\right)\right\|_{0} \leq C h^{2}|u|_{4},
$$

provided that $u \in H^{4}(\Omega) \cap H_{0}^{2}(\Omega)$.

Proof. The proof is the same as that for Theorem 3.2.

\section{The Basis Functions of the Two New Elements in $2 \mathrm{D}$}

In this section, we give explicitly the basis functions on each element $K$.

\subsection{The basis functions for the $P_{3}+2 B_{4}$ element}

The first six nodal basis functions are associated to the 0th and 1st moments of normal derivative on three edges of element $K$.

$$
\begin{aligned}
& \varphi_{1,1}(\mathbf{x})=r_{1}\left(4\left(\lambda_{2}^{2} \lambda_{3}-\lambda_{2} \lambda_{3}^{2}\right)-2\left(\lambda_{3}^{2} \lambda_{1}-\lambda_{3} \lambda_{1}^{2}\right)-2 \lambda_{1}^{2} \lambda_{2} \lambda_{3}-22 \lambda_{1} \lambda_{2}^{2} \lambda_{3}+18 \lambda_{1} \lambda_{2} \lambda_{3}^{2}\right), \\
& \varphi_{1,2}(\mathbf{x})=r_{1}\left(2\left(\lambda_{1}^{2} \lambda_{2}-\lambda_{1} \lambda_{2}^{2}\right)-4\left(\lambda_{2}^{2} \lambda_{3}-\lambda_{2} \lambda_{3}^{2}\right)-2 \lambda_{1}^{2} \lambda_{2} \lambda_{3}+18 \lambda_{1} \lambda_{2}^{2} \lambda_{3}-22 \lambda_{1} \lambda_{2} \lambda_{3}^{2}\right), \\
& \varphi_{2,1}(\mathbf{x})=r_{2}\left(2\left(\lambda_{2}^{2} \lambda_{3}-\lambda_{2} \lambda_{3}^{2}\right)-4\left(\lambda_{3}^{2} \lambda_{1}-\lambda_{3} \lambda_{1}^{2}\right)-22 \lambda_{1}^{2} \lambda_{2} \lambda_{3}-2 \lambda_{1} \lambda_{2}^{2} \lambda_{3}+18 \lambda_{1} \lambda_{2} \lambda_{3}^{2}\right), \\
& \varphi_{2,2}(\mathbf{x})=r_{2}\left(-2\left(\lambda_{1}^{2} \lambda_{2}-\lambda_{1} \lambda_{2}^{2}\right)+4\left(\lambda_{3}^{2} \lambda_{1}-\lambda_{3} \lambda_{1}^{2}\right)+18 \lambda_{1}^{2} \lambda_{2} \lambda_{3}-2 \lambda_{1} \lambda_{2}^{2} \lambda_{3}-22 \lambda_{1} \lambda_{2} \lambda_{3}^{2}\right), \\
& \varphi_{3,1}(\mathbf{x})=r_{3}\left(4\left(\lambda_{1}^{2} \lambda_{2}-\lambda_{1} \lambda_{2}^{2}\right)-2\left(\lambda_{2}^{2} \lambda_{3}-\lambda_{2} \lambda_{3}^{2}\right)-22 \lambda_{1}^{2} \lambda_{2} \lambda_{3}+18 \lambda_{1} \lambda_{2}^{2} \lambda_{3}-2 \lambda_{1} \lambda_{2} \lambda_{3}^{2}\right), \\
& \varphi_{3,2}(\mathbf{x})=r_{3}\left(-4\left(\lambda_{1}^{2} \lambda_{2}-\lambda_{1} \lambda_{2}^{2}\right)+2\left(\lambda_{3}^{2} \lambda_{1}-\lambda_{3} \lambda_{1}^{2}\right)+18 \lambda_{1}^{2} \lambda_{2} \lambda_{3}-22 \lambda_{1} \lambda_{2}^{2} \lambda_{3}-2 \lambda_{1} \lambda_{2} \lambda_{3}^{2}\right) .
\end{aligned}
$$

These six nodal basis functions satisfy the usual orthogonal conditions in the sense that

$$
\frac{1}{\left|e_{k}\right|} \int_{e_{k}} \frac{\partial \varphi_{l, i}}{\partial \mathbf{n}_{k}} \lambda_{e_{k}}^{(j)} d s=\delta_{l, k} \delta_{i, j}, \quad l, k=1,2,3, \quad i, j=1,2
$$

Here and in the following, $\lambda_{e_{k}}^{(j)}, j=1,2$, are the edge barycenter coordinates on $e_{k}$, for instance, $\lambda_{e_{1}}^{(1)}=\lambda_{2}, \lambda_{e_{1}}^{(2)}=\lambda_{3}$, on $e_{1}$. Note that the six nodal basis functions vanish for the remaining degrees of freedom in (3.1a), namely,

$$
\varphi_{l, i}\left(\mathbf{x}_{k}\right)=0, \quad \int_{e_{k}} \varphi_{l, i} d s=0, \quad l, k=1,2,3, \quad i=1,2 .
$$


Using the edge barycenter coordinates, these six basis functions can be expressed in a unifying way:

$$
\begin{array}{r}
\varphi_{l, i}(\mathbf{x})=r_{l}\left(4\left(\left(\lambda_{e_{l}}^{(i)}\right)^{2} \lambda_{e_{l}}^{(i+1)}-\left(\lambda_{e_{l}}^{(i+1)}\right)^{2} \lambda_{e_{l}}^{(i)}\right)-2\left(\left(\lambda_{e_{l}}^{(i+1)}\right)^{2} \lambda_{l}-\lambda_{l}^{2} \lambda_{e_{l}}^{(i+1)}\right)\right. \\
\left.-\lambda_{1} \lambda_{2} \lambda_{3}\left(22 \lambda_{e_{l}}^{(i)}-18 \lambda_{e_{l}}^{(i+1)}+2 \lambda_{l}\right)\right), \quad l=1,2,3, \quad i=1,2 .
\end{array}
$$

The second three nodal basis functions are associated to the 0th moment of function on three edges of element $K$.

$$
\begin{aligned}
& \phi_{1}(\mathbf{x})=12 \lambda_{2} \lambda_{3}^{2}+\left(\mathbf{n}_{1}\right)^{T}\left(\left(\frac{\mathbf{n}_{2}}{r_{2}}+2 \frac{\mathbf{n}_{3}}{r_{3}}\right) \varphi_{1,1}(\mathbf{x})+\left(3 \frac{\mathbf{n}_{2}}{r_{2}}+2 \frac{\mathbf{n}_{3}}{r_{3}}\right) \varphi_{1,2}(\mathbf{x})\right)+\frac{\varphi_{2,1}(\mathbf{x})+3 \varphi_{2,2}(\mathbf{x})}{r_{2}}, \\
& \phi_{2}(\mathbf{x})=12 \lambda_{3} \lambda_{1}^{2}+\left(\mathbf{n}_{2}\right)^{T}\left(\left(2 \frac{\mathbf{n}_{1}}{r_{1}}+3 \frac{\mathbf{n}_{3}}{r_{3}}\right) \varphi_{2,1}(\mathbf{x})+\left(2 \frac{\mathbf{n}_{1}}{r_{1}}+\frac{\mathbf{n}_{3}}{r_{3}}\right) \varphi_{2,2}(\mathbf{x})\right)+\frac{3 \varphi_{3,1}(\mathbf{x})+\varphi_{3,2}(\mathbf{x})}{r_{3}}, \\
& \phi_{3}(\mathbf{x})=12 \lambda_{1} \lambda_{2}^{2}+\left(\mathbf{n}_{3}\right)^{T}\left(\left(\frac{\mathbf{n}_{1}}{r_{1}}+2 \frac{\mathbf{n}_{2}}{r_{2}}\right) \varphi_{3,1}(\mathbf{x})+\left(3 \frac{\mathbf{n}_{1}}{r_{1}}+2 \frac{\mathbf{n}_{2}}{r_{2}}\right) \varphi_{3,2}(\mathbf{x})\right)+\frac{3 \varphi_{1,1}(\mathbf{x})+\varphi_{1,2}(\mathbf{x})}{r_{1}} .
\end{aligned}
$$

These three nodal basis functions satisfy the usual orthogonal conditions in the sense that

$$
\frac{1}{\left|e_{k}\right|} \int_{e_{k}} \phi_{l}(\mathbf{x}) d s=\delta_{l, k}, \quad l, k=1,2,3
$$

and vanish for the remaining degrees of freedom in (3.1a), namely,

$$
\frac{1}{\left|e_{k}\right|} \int_{e_{k}} \frac{\partial \phi_{l}(\mathbf{x})}{\partial \mathbf{n}_{k}} \lambda_{e_{k}}^{(j)} d s=0, \quad \phi_{l}\left(\mathbf{x}_{k}\right)=0, \quad l, k=1,2,3, \quad j=1,2 .
$$

The last three nodal basis functions are associated to the value of function at three vertices of element $K$.

$$
\begin{aligned}
& \theta_{1}(\mathbf{x})=\lambda_{1}^{3}+\left(\mathbf{n}_{1}\right)^{T} \mathbf{n}_{2}\left(\frac{3}{4} \varphi_{2,1}(\mathbf{x})+\frac{1}{4} \varphi_{2,2}(\mathbf{x})\right) / r_{1} \\
&+\left(\mathbf{n}_{1}\right)^{T} \mathbf{n}_{3}\left(\frac{3}{4} \varphi_{3,1}(\mathbf{x})+\frac{1}{4} \varphi_{3,2}(\mathbf{x})\right) / r_{1}-\frac{1}{4}\left(\phi_{2}(\mathbf{x})+\phi_{3}(\mathbf{x})\right), \\
& \theta_{2}(\mathbf{x})=\lambda_{2}^{3}+\left(\mathbf{n}_{2}\right)^{T} \mathbf{n}_{1}\left(\frac{3}{4} \varphi_{1,1}(\mathbf{x})+\frac{1}{4} \varphi_{1,2}(\mathbf{x})\right) / r_{2} \\
&+\left(\mathbf{n}_{2}\right)^{T} \mathbf{n}_{3}\left(\frac{1}{4} \varphi_{3,1}(\mathbf{x})+\frac{3}{4} \varphi_{3,2}(\mathbf{x})\right) / r_{2}-\frac{1}{4}\left(\phi_{1}(\mathbf{x})+\phi_{3}(\mathbf{x})\right), \\
& \theta_{3}(\mathbf{x})=\lambda_{3}^{3}+\left(\mathbf{n}_{3}\right)^{T} \mathbf{n}_{1}\left(\frac{1}{4} \varphi_{1,1}(\mathbf{x})+\frac{3}{4} \varphi_{1,2}(\mathbf{x})\right) / r_{3} \\
&+\left(\mathbf{n}_{3}\right)^{T} \mathbf{n}_{2}\left(\frac{1}{4} \varphi_{2,1}(\mathbf{x})+\frac{3}{4} \varphi_{2,2}(\mathbf{x})\right) / r_{3}-\frac{1}{4}\left(\phi_{1}(\mathbf{x})+\phi_{2}(\mathbf{x})\right) .
\end{aligned}
$$

These three nodal basis functions satisfy the usual orthogonal conditions in the sense that

$$
\theta_{l}\left(\mathbf{x}_{k}\right)=\delta_{l, k}, \quad l, k=1,2,3,
$$

and vanish for the remaining degrees of freedom in (3.1a), namely,

$$
\frac{1}{\left|e_{k}\right|} \int_{e_{k}} \frac{\partial \theta_{l}(\mathbf{x})}{\partial \mathbf{n}_{k}} \lambda_{e_{k}}^{(j)} d s=0, \quad \frac{1}{\left|e_{k}\right|} \int_{e_{k}} \theta_{l}(\mathbf{x}) d s=0, \quad l, k=1,2,3, \quad j=1,2 .
$$




\subsection{The basis functions for the $P_{4}$ element}

The first six nodal basis functions are associated to the normal derivatives.

$$
\begin{aligned}
& \varphi_{1,1}(\mathbf{x})=r_{1}\left(-\frac{3}{2}\left(2 \lambda_{1}^{3} \lambda_{2}-3 \lambda_{1}^{2} \lambda_{2}^{2}\right)-\left(2 \lambda_{1} \lambda_{2}^{3}-3 \lambda_{1}^{2} \lambda_{2}^{2}\right)+\left(2 \lambda_{2}^{3} \lambda_{3}-3 \lambda_{2}^{2} \lambda_{3}^{2}\right)\right. \\
& -\frac{1}{2}\left(2 \lambda_{2} \lambda_{3}^{3}-3 \lambda_{2}^{2} \lambda_{3}^{2}\right)+\frac{1}{2}\left(2 \lambda_{3}^{3} \lambda_{1}-3 \lambda_{3}^{2} \lambda_{1}^{2}\right)-\frac{1}{2}\left(2 \lambda_{3} \lambda_{1}^{3}-3 \lambda_{3}^{2} \lambda_{1}^{2}\right) \\
& \left.+9 \lambda_{1}^{2} \lambda_{2} \lambda_{3}-12 \lambda_{1} \lambda_{2}^{2} \lambda_{3}+3 \lambda_{1} \lambda_{2} \lambda_{3}^{2}\right), \\
& \varphi_{1,2}(\mathbf{x})=r_{1}\left(-\frac{1}{2}\left(2 \lambda_{1}^{3} \lambda_{2}-3 \lambda_{1}^{2} \lambda_{2}^{2}\right)+\frac{1}{2}\left(2 \lambda_{1} \lambda_{2}^{3}-3 \lambda_{1}^{2} \lambda_{2}^{2}\right)-\frac{1}{2}\left(2 \lambda_{2}^{3} \lambda_{3}-3 \lambda_{2}^{2} \lambda_{3}^{2}\right)\right. \\
& +\left(2 \lambda_{2} \lambda_{3}^{3}-3 \lambda_{2}^{2} \lambda_{3}^{2}\right)-\left(2 \lambda_{3}^{3} \lambda_{1}-3 \lambda_{3}^{2} \lambda_{1}^{2}\right)-\frac{3}{2}\left(2 \lambda_{3} \lambda_{1}^{3}-3 \lambda_{3}^{2} \lambda_{1}^{2}\right) \\
& \left.+9 \lambda_{1}^{2} \lambda_{2} \lambda_{3}+3 \lambda_{1} \lambda_{2}^{2} \lambda_{3}-12 \lambda_{1} \lambda_{2} \lambda_{3}^{2}\right), \\
& \varphi_{2,1}(\mathbf{x})=r_{2}\left(-\left(2 \lambda_{1}^{3} \lambda_{2}-3 \lambda_{1}^{2} \lambda_{2}^{2}\right)-\frac{3}{2}\left(2 \lambda_{1} \lambda_{2}^{3}-3 \lambda_{1}^{2} \lambda_{2}^{2}\right)-\frac{1}{2}\left(2 \lambda_{2}^{3} \lambda_{3}-3 \lambda_{2}^{2} \lambda_{3}^{2}\right)\right. \\
& +\frac{1}{2}\left(2 \lambda_{2} \lambda_{3}^{3}-3 \lambda_{2}^{2} \lambda_{3}^{2}\right)-\frac{1}{2}\left(2 \lambda_{3}^{3} \lambda_{1}-3 \lambda_{3}^{2} \lambda_{1}^{2}\right)+\left(2 \lambda_{3} \lambda_{1}^{3}-3 \lambda_{3}^{2} \lambda_{1}^{2}\right) \\
& \left.-12 \lambda_{1}^{2} \lambda_{2} \lambda_{3}+9 \lambda_{1} \lambda_{2}^{2} \lambda_{3}+3 \lambda_{1} \lambda_{2} \lambda_{3}^{2}\right), \\
& \varphi_{2,2}(\mathbf{x})=r_{2}\left(\frac{1}{2}\left(2 \lambda_{1}^{3} \lambda_{2}-3 \lambda_{1}^{2} \lambda_{2}^{2}\right)-\frac{1}{2}\left(2 \lambda_{1} \lambda_{2}^{3}-3 \lambda_{1}^{2} \lambda_{2}^{2}\right)-\frac{3}{2}\left(2 \lambda_{2}^{3} \lambda_{3}-3 \lambda_{2}^{2} \lambda_{3}^{2}\right)\right. \\
& -\left(2 \lambda_{2} \lambda_{3}^{3}-3 \lambda_{2}^{2} \lambda_{3}^{2}\right)+\left(2 \lambda_{3}^{3} \lambda_{1}-3 \lambda_{3}^{2} \lambda_{1}^{2}\right)-\frac{1}{2}\left(2 \lambda_{3} \lambda_{1}^{3}-3 \lambda_{3}^{2} \lambda_{1}^{2}\right) \\
& \left.+3 \lambda_{1}^{2} \lambda_{2} \lambda_{3}+9 \lambda_{1} \lambda_{2}^{2} \lambda_{3}-12 \lambda_{1} \lambda_{2} \lambda_{3}^{2}\right), \\
& \varphi_{3,1}(\mathbf{x})=r_{3}\left(\left(2 \lambda_{1}^{3} \lambda_{2}-3 \lambda_{1}^{2} \lambda_{2}^{2}\right)-\frac{1}{2}\left(2 \lambda_{1} \lambda_{2}^{3}-3 \lambda_{1}^{2} \lambda_{2}^{2}\right)+\frac{1}{2}\left(2 \lambda_{2}^{3} \lambda_{3}-3 \lambda_{2}^{2} \lambda_{3}^{2}\right)\right. \\
& -\frac{1}{2}\left(2 \lambda_{2} \lambda_{3}^{3}-3 \lambda_{2}^{2} \lambda_{3}^{2}\right)-\frac{3}{2}\left(2 \lambda_{3}^{3} \lambda_{1}-3 \lambda_{3}^{2} \lambda_{1}^{2}\right)-\left(2 \lambda_{3} \lambda_{1}^{3}-3 \lambda_{3}^{2} \lambda_{1}^{2}\right) \\
& \left.-12 \lambda_{1}^{2} \lambda_{2} \lambda_{3}+3 \lambda_{1} \lambda_{2}^{2} \lambda_{3}+9 \lambda_{1} \lambda_{2} \lambda_{3}^{2}\right), \\
& \varphi_{3,2}(\mathbf{x})=r_{3}\left(-\frac{1}{2}\left(2 \lambda_{1}^{3} \lambda_{2}-3 \lambda_{1}^{2} \lambda_{2}^{2}\right)+\left(2 \lambda_{1} \lambda_{2}^{3}-3 \lambda_{1}^{2} \lambda_{2}^{2}\right)-\left(2 \lambda_{2}^{3} \lambda_{3}-3 \lambda_{2}^{2} \lambda_{3}^{2}\right)\right. \\
& -\frac{3}{2}\left(2 \lambda_{2} \lambda_{3}^{3}-3 \lambda_{2}^{2} \lambda_{3}^{2}\right)-\frac{1}{2}\left(2 \lambda_{3}^{3} \lambda_{1}-3 \lambda_{3}^{2} \lambda_{1}^{2}\right)+\frac{1}{2}\left(2 \lambda_{3} \lambda_{1}^{3}-3 \lambda_{3}^{2} \lambda_{1}^{2}\right) \\
& \left.+3 \lambda_{1}^{2} \lambda_{2} \lambda_{3}-12 \lambda_{1} \lambda_{2}^{2} \lambda_{3}+9 \lambda_{1} \lambda_{2} \lambda_{3}^{2}\right) \text {. }
\end{aligned}
$$

Using the edge barycenter coordinates, the above six basis functions can be expressed in a unifying way:

$$
\begin{aligned}
\varphi_{l, i}(\mathbf{x})=r_{l}( & -\frac{3}{2}\left(2 \lambda_{l}^{3} \lambda_{e_{l}}^{(i)}-3 \lambda_{l}^{2}\left(\lambda_{e_{l}}^{(i)}\right)^{2}\right)-\left(2\left(\lambda_{e_{l}}^{(i)}\right)^{3} \lambda_{l}-3 \lambda_{l}^{2}\left(\lambda_{e_{l}}^{(i)}\right)^{2}\right) \\
& +\left(2 \lambda_{e_{l}}^{(i+1)}\left(\lambda_{e_{l}}^{(i)}\right)^{3}-3\left(\lambda_{e_{l}}^{(i+1)}\right)^{2}\left(\lambda_{e_{l}}^{(i)}\right)^{2}\right)-\frac{1}{2}\left(2 \lambda_{e_{l}}^{(i)}\left(\lambda_{e_{l}}^{(i+1)}\right)^{3}-3\left(\lambda_{e_{l}}^{(i+1)}\right)^{2}\left(\lambda_{e_{l}}^{(i)}\right)^{2}\right) \\
& +\frac{1}{2}\left(2 \lambda_{l}\left(\lambda_{e_{l}}^{(i+1)}\right)^{3}-3\left(\lambda_{e_{l}}^{(i+1)}\right)^{2} \lambda_{l}^{2}\right)-\frac{1}{2}\left(2 \lambda_{l}^{3} \lambda_{e_{l}}^{(i+1)}-3\left(\lambda_{e_{l}}^{(i+1)}\right)^{2} \lambda_{l}^{2}\right) \\
& \left.+\lambda_{1} \lambda_{2} \lambda_{3}\left(9 \lambda_{l}-12 \lambda_{e_{l}}^{(i)}+3 \lambda_{e_{l}}^{(i+1)}\right)\right), \quad l=1,2,3, \quad i=1,2 .
\end{aligned}
$$


The second three nodal basis functions are associated to the 0th and 1st moments of function on element $K$.

$$
\begin{aligned}
& \psi_{1}(\mathbf{x})=\left(120\left(2 \lambda_{1}^{3} \lambda_{2}-3 \lambda_{1}^{2} \lambda_{2}^{2}\right)-90\left(2 \lambda_{1} \lambda_{2}^{3}-3 \lambda_{1}^{2} \lambda_{2}^{2}\right)-90\left(2 \lambda_{2}^{3} \lambda_{3}-3 \lambda_{2}^{2} \lambda_{3}^{2}\right)\right. \\
& -90\left(2 \lambda_{2} \lambda_{3}^{3}-3 \lambda_{2}^{2} \lambda_{3}^{2}\right)-90\left(2 \lambda_{3}^{3} \lambda_{1}-3 \lambda_{3}^{2} \lambda_{1}^{2}\right)+120\left(2 \lambda_{3} \lambda_{1}^{3}-3 \lambda_{3}^{2} \lambda_{1}^{2}\right) \\
& \left.-720 \lambda_{1}^{2} \lambda_{2} \lambda_{3}+540 \lambda_{1} \lambda_{2}^{2} \lambda_{3}+540 \lambda_{1} \lambda_{2} \lambda_{3}^{2}\right) \text {, } \\
& \psi_{2}(\mathbf{x})=\left(-90\left(2 \lambda_{1}^{3} \lambda_{2}-3 \lambda_{1}^{2} \lambda_{2}^{2}\right)+120\left(2 \lambda_{1} \lambda_{2}^{3}-3 \lambda_{1}^{2} \lambda_{2}^{2}\right)+120\left(2 \lambda_{2}^{3} \lambda_{3}-3 \lambda_{2}^{2} \lambda_{3}^{2}\right)\right. \\
& -90\left(2 \lambda_{2} \lambda_{3}^{3}-3 \lambda_{2}^{2} \lambda_{3}^{2}\right)-90\left(2 \lambda_{3}^{3} \lambda_{1}-3 \lambda_{3}^{2} \lambda_{1}^{2}\right)-90\left(2 \lambda_{3} \lambda_{1}^{3}-3 \lambda_{3}^{2} \lambda_{1}^{2}\right) \\
& \left.+540 \lambda_{1}^{2} \lambda_{2} \lambda_{3}-720 \lambda_{1} \lambda_{2}^{2} \lambda_{3}+540 \lambda_{1} \lambda_{2} \lambda_{3}^{2}\right) \text {, } \\
& \psi_{3}(\mathbf{x})=\left(-90\left(2 \lambda_{1}^{3} \lambda_{2}-3 \lambda_{1}^{2} \lambda_{2}^{2}\right)-90\left(2 \lambda_{1} \lambda_{2}^{3}-3 \lambda_{1}^{2} \lambda_{2}^{2}\right)-90\left(2 \lambda_{2}^{3} \lambda_{3}-3 \lambda_{2}^{2} \lambda_{3}^{2}\right)\right. \\
& +120\left(2 \lambda_{2} \lambda_{3}^{3}-3 \lambda_{2}^{2} \lambda_{3}^{2}\right)+120\left(2 \lambda_{3}^{3} \lambda_{1}-3 \lambda_{3}^{2} \lambda_{1}^{2}\right)-90\left(2 \lambda_{3} \lambda_{1}^{3}-3 \lambda_{3}^{2} \lambda_{1}^{2}\right) \\
& \left.+540 \lambda_{1}^{2} \lambda_{2} \lambda_{3}+540 \lambda_{1} \lambda_{2}^{2} \lambda_{3}-720 \lambda_{1} \lambda_{2} \lambda_{3}^{2}\right) \text {. }
\end{aligned}
$$

The above three basis functions can be expressed in a unifying way:

$$
\begin{aligned}
\psi_{i}(\mathbf{x})=( & 120\left(2 \lambda_{i}^{3} \lambda_{j}-3 \lambda_{i}^{2} \lambda_{j}^{2}\right)+120\left(2 \lambda_{k} \lambda_{i}^{3}-3 \lambda_{k}^{2} \lambda_{i}^{2}\right)-90\left(2 \lambda_{i} \lambda_{j}^{3}-3 \lambda_{i}^{2} \lambda_{j}^{2}\right) \\
& -90\left(2 \lambda_{i} \lambda_{k}^{3}-3 \lambda_{k}^{2} \lambda_{i}^{2}\right)-90\left(2 \lambda_{k} \lambda_{j}^{3}-3 \lambda_{k}^{2} \lambda_{j}^{2}\right)-90\left(2 \lambda_{k}^{3} \lambda_{j}-3 \lambda_{j}^{2} \lambda_{k}^{2}\right) \\
& \left.-\lambda_{1} \lambda_{2} \lambda_{3}\left(720 \lambda_{i}-540 \lambda_{j}-540 \lambda_{k}\right)\right)
\end{aligned}
$$

where $(i, j, k)$ is a permutation of $(1,2,3)$. The third three nodal basis functions are associated to the 0th moment of function on three edges of element $K$.

$$
\begin{gathered}
\phi_{1}(\mathbf{x})=30 \lambda_{2}^{2} \lambda_{3}^{2}+\left(\mathbf{n}_{1}\right)^{T}\left(\left(2 \frac{\mathbf{n}_{2}}{r_{2}}+3 \frac{\mathbf{n}_{3}}{r_{3}}\right) \varphi_{1,1}(\mathbf{x})+\left(3 \frac{\mathbf{n}_{2}}{r_{2}}+2 \frac{\mathbf{n}_{3}}{r_{3}}\right) \varphi_{1,2}(\mathbf{x})\right) \\
-\frac{1}{42} \psi_{1}(\mathbf{x})-\frac{1}{14} \psi_{2}(\mathbf{x})-\frac{1}{14} \psi_{3}(\mathbf{x}), \\
\phi_{2}(\mathbf{x})=30 \lambda_{1}^{2} \lambda_{3}^{2}+\left(\mathbf{n}_{2}\right)^{T}\left(\left(2 \frac{\mathbf{n}_{1}}{r_{1}}+3 \frac{\mathbf{n}_{3}}{r_{3}}\right) \varphi_{2,1}(\mathbf{x})+\left(3 \frac{\mathbf{n}_{1}}{r_{1}}+2 \frac{\mathbf{n}_{3}}{r_{3}}\right) \varphi_{2,2}(\mathbf{x})\right) \\
-\frac{1}{14} \psi_{1}(\mathbf{x})-\frac{1}{42} \psi_{2}(\mathbf{x})-\frac{1}{14} \psi_{3}(\mathbf{x}), \\
\phi_{3}(\mathbf{x})=30 \lambda_{1}^{2} \lambda_{2}^{2}+\left(\mathbf{n}_{3}\right)^{T}\left(\left(2 \frac{\mathbf{n}_{1}}{r_{1}}+3 \frac{\mathbf{n}_{2}}{r_{2}}\right) \varphi_{3,1}(\mathbf{x})+\left(3 \frac{\mathbf{n}_{1}}{r_{1}}+2 \frac{\mathbf{n}_{2}}{r_{2}}\right) \varphi_{3,2}(\mathbf{x})\right) \\
-\frac{1}{14} \psi_{1}(\mathbf{x})-\frac{1}{14} \psi_{2}(\mathbf{x})-\frac{1}{42} \psi_{3}(\mathbf{x}) .
\end{gathered}
$$

The last three nodal basis functions are associated to the value of function at three vertices 
of element $K$.

$$
\begin{aligned}
\theta_{1}(\mathbf{x})=\lambda_{1}^{4}+ & \frac{\mathbf{n}_{1}^{T} \mathbf{n}_{2}}{5 r_{1}}\left(4 \varphi_{2,1}(\mathbf{x})+\varphi_{2,2}(\mathbf{x})\right)+\frac{\mathbf{n}_{1}^{T} \mathbf{n}_{3}}{5 r_{1}}\left(4 \varphi_{3,1}(\mathbf{x})+\varphi_{3,2}(\mathbf{x})\right) \\
& -\frac{1}{5}\left(\phi_{2}(\mathbf{x})+\phi_{3}(\mathbf{x})\right)-\frac{1}{42} \psi_{1}(\mathbf{x})-\frac{1}{210} \psi_{2}(\mathbf{x})-\frac{1}{210} \psi_{3}(\mathbf{x}), \\
\theta_{2}(\mathbf{x})=\lambda_{2}^{4}+ & \frac{\mathbf{n}_{1}^{T} \mathbf{n}_{2}}{5 r_{2}}\left(4 \varphi_{1,1}(\mathbf{x})+\varphi_{1,2}(\mathbf{x})\right)+\frac{\mathbf{n}_{2}^{T} \mathbf{n}_{3}}{5 r_{2}}\left(\varphi_{3,1}(\mathbf{x})+4 \varphi_{3,2}(\mathbf{x})\right) \\
& -\frac{1}{5}\left(\phi_{1}(\mathbf{x})+\phi_{3}(\mathbf{x})\right)-\frac{1}{210} \psi_{1}(\mathbf{x})-\frac{1}{42} \psi_{2}(\mathbf{x})-\frac{1}{210} \psi_{3}(\mathbf{x}), \\
\theta_{3}(\mathbf{x})=\lambda_{3}^{4}+ & \frac{\mathbf{n}_{1}^{T} \mathbf{n}_{3}}{5 r_{3}}\left(\varphi_{1,1}(\mathbf{x})+4 \varphi_{1,2}(\mathbf{x})\right)+\frac{\mathbf{n}_{2}^{T} \mathbf{n}_{3}}{5 r_{3}}\left(\varphi_{2,1}(\mathbf{x})+4 \varphi_{2,2}(\mathbf{x})\right) \\
& -\frac{1}{5}\left(\phi_{1}(\mathbf{x})+\phi_{2}(\mathbf{x})\right)-\frac{1}{210} \psi_{1}(\mathbf{x})-\frac{1}{210} \psi_{2}(\mathbf{x})-\frac{1}{42} \psi_{3}(\mathbf{x}) .
\end{aligned}
$$

For these thirty nodal basis functions, the twelve basis functions $\varphi_{1, i}(\mathbf{x}), \phi_{l}(\mathbf{x})$ and $\theta_{l}(\mathbf{x})$ are similarly defined as those for the enriched $P_{3}$ element in the previous subsection. In addition, they vanish for the following three conditions

$$
\int_{K}(\cdot) \lambda_{l} d x=0, \quad l=1,2,3 .
$$

Compared to the enriched $P_{3}$ element, there are three more basis functions $\psi_{l}(\mathbf{x})$ which are associated to the three moments on element $K$ in (5.1) and satisfy the following orthogonal conditions

$$
\frac{1}{|K|} \int_{K} \psi_{l}(\mathbf{x}) \lambda_{k} d x=\delta_{l, k}, \quad l, k=1,2,3 .
$$

Moreover $\psi_{l}(\mathbf{x})$ vanish for the remaining degrees of freedom in (4.1).

\section{A $P_{3}+8 B_{4}$ Element in $3 \mathrm{D}$}

On a tetrahedral grid $\mathcal{T}_{h}=\{K\}$ of $\Omega$, the finite element shape functions space can be chosen as

$$
P_{3}^{+}(K):=P_{3}(K)+\operatorname{span}\left\{\lambda_{1}^{2} \lambda_{2} \lambda_{4}, \lambda_{1}^{2} \lambda_{3} \lambda_{4}, \lambda_{2}^{2} \lambda_{3} \lambda_{4}, \lambda_{1} \lambda_{2}^{2} \lambda_{3}, \lambda_{2} \lambda_{3}^{2} \lambda_{4}, \lambda_{1} \lambda_{2} \lambda_{3}^{2}, \lambda_{1} \lambda_{2} \lambda_{4}^{2}, \lambda_{1} \lambda_{3} \lambda_{4}^{2}\right\},
$$

where $P_{3}(K)$ denotes the space of $P_{3}$ polynomials, $\lambda_{i}$ is the barycentric coordinate for vertex $\mathbf{x}_{i}$, a linear polynomial vanishing on the face triangle opposite to the vertex.

\subsection{The degrees of freedom}

The dual basis of the finite element space, i.e., the degrees of freedom of the finite element, is

$$
\begin{cases}\mathbb{E}_{j, k}(\cdot):=\frac{1}{\left|e_{j}\right|} \int_{e_{j}}(\cdot) \lambda_{e_{j}}^{(k)} d s & \text { on edge } e_{j}, j=1, \cdots, 6, k=1,2, \\ \mathbb{F}_{j}(\cdot):=\frac{1}{\left|F_{j}\right|} \int_{F_{j}}(\cdot) d f & \text { on face } F_{j}, j=1, \cdots, 4, \\ \mathbb{N}_{j, k}(\cdot):=\frac{1}{\left|F_{j}\right|} \int_{F_{j}} \partial_{\mathbf{n}_{j}}(\cdot) \lambda_{F_{j}}^{(k)} d f & \text { on face } F_{j}, j=1, \cdots, 4, k=1,2,3 .\end{cases}
$$


Here $\lambda_{e_{j}}^{(k)}$ are two 1D barycentric coordinates on the edge $e_{j}, \lambda_{F_{j}}^{(k)}$ are three 2D barycenter coordinates on the face triangle $F_{j}$, in the sense that $\lambda_{F_{1}}^{(1)}=\lambda_{2}, \lambda_{F_{1}}^{(2)}=\lambda_{3}$ and $\lambda_{F_{1}}^{(3)}=\lambda_{4}$. In addition, let $r_{j}$ be the distance from $\mathbf{x}_{j}$ to face $F_{j}$. The global finite element space is defined by

$$
\begin{aligned}
& V_{h, H Z 28}:=\left\{v \in L^{2}(\Omega)|v|_{K} \in P_{3}^{+}(K), \int_{e} v p_{1} d s \text { is continuous around internal edges of } \mathcal{T}_{h},\right. \\
& \int_{F} v d f \& \int_{F} \partial_{\mathbf{n}} v p_{1} d f \quad \text { are continuous across internal face triangles of } \mathcal{T}_{h} \\
& \int_{e} v p_{1} d s=\int_{F} v d f=\int_{F} \partial_{\mathbf{n}} v p_{1} d f=0 \\
& \text { at boundary edges and face triangles of } \left.\mathcal{T}_{h}\right\} \text {, }
\end{aligned}
$$

where $p_{1}$ is a linear function on an edge or a face triangle.

Theorem 6.1. The 28 degrees of freedom in (6.1) uniquely determine a polynomial of $P_{3}^{+}(K)$.

Proof. Let $u \in P_{3}^{+}(K)$ such that the 28 degrees of freedom of $u$ in (6.1) have zero value. Since we use only 2 face bubbles instead of 3 face bubbles, the nodal basis on a general element depends on the numbering of vertex. First we list twelve functions which have zero edge moments and face value moments. In fact, these functions vanish on all edges and faces, except one face where only the average vanishes.

$$
\begin{array}{ll}
b_{1}=\lambda_{1} \lambda_{2} \lambda_{4}\left(3 \lambda_{1}-1\right), & b_{2}=\lambda_{1} \lambda_{3} \lambda_{4}\left(3 \lambda_{1}-1\right), \\
b_{3}=\lambda_{2} \lambda_{1} \lambda_{3}\left(3 \lambda_{2}-1\right), & b_{4}=\lambda_{2} \lambda_{3} \lambda_{4}\left(3 \lambda_{2}-1\right), \\
b_{5}=\lambda_{3} \lambda_{1} \lambda_{2}\left(3 \lambda_{3}-1\right), & b_{6}=\lambda_{3} \lambda_{2} \lambda_{4}\left(3 \lambda_{3}-1\right), \\
b_{7}=\lambda_{4} \lambda_{1} \lambda_{2}\left(3 \lambda_{4}-1\right), & b_{8}=\lambda_{4} \lambda_{1} \lambda_{3}\left(3 \lambda_{4}-1\right) .
\end{array}
$$

Before we find the rest 4 basis functions which have 16 vanishing edge and face moments, we find 16 dual-basis functions (they do not have vanishing normal derivative moments and they are not unique).

$$
\begin{aligned}
& b_{13}=12 \lambda_{1} \lambda_{2}\left(5 \lambda_{1}-2\right), \quad b_{14}=12 \lambda_{1} \lambda_{2}\left(5 \lambda_{2}-2\right), \quad b_{15}=12 \lambda_{1} \lambda_{3}\left(5 \lambda_{1}-2\right), \\
& b_{16}=12 \lambda_{1} \lambda_{3}\left(5 \lambda_{3}-2\right), \quad b_{17}=12 \lambda_{1} \lambda_{4}\left(5 \lambda_{1}-2\right), \quad b_{18}=12 \lambda_{1} \lambda_{4}\left(5 \lambda_{4}-2\right), \\
& b_{19}=12 \lambda_{2} \lambda_{3}\left(5 \lambda_{2}-2\right), \quad b_{20}=12 \lambda_{2} \lambda_{3}\left(5 \lambda_{3}-2\right), \quad b_{21}=12 \lambda_{2} \lambda_{4}\left(5 \lambda_{2}-2\right), \\
& b_{22}=12 \lambda_{2} \lambda_{4}\left(5 \lambda_{4}-2\right), \quad b_{23}=12 \lambda_{3} \lambda_{4}\left(5 \lambda_{3}-2\right), \quad b_{24}=12 \lambda_{3} \lambda_{4}\left(5 \lambda_{4}-2\right), \\
& b_{25}=60 \lambda_{1} \lambda_{2} \lambda_{3}, \quad b_{26}=60 \lambda_{1} \lambda_{2} \lambda_{4}, \quad b_{27}=60 \lambda_{1} \lambda_{3} \lambda_{4}, \quad b_{28}=60 \lambda_{2} \lambda_{3} \lambda_{4} .
\end{aligned}
$$

For the first 16 dual basis functionals, we have

$$
\begin{aligned}
& \mathbb{E}_{j, k}\left(b_{i+12}\right)=\delta_{i, 2(j-1)+k}, \quad \mathbb{F}_{m}\left(b_{i+12}\right)=0, \quad 1 \leq j \leq 6, \quad k=1,2, \quad 1 \leq m \leq 4, \quad 1 \leq i \leq 12, \\
& \mathbb{F}_{m}\left(b_{i+24}\right)=\delta_{i, m}, \quad \mathbb{E}_{j, k}\left(b_{i+24}\right)=0, \quad 1 \leq j \leq 6, \quad k=1,2, \quad 1 \leq m \leq 4, \quad 1 \leq i \leq 4
\end{aligned}
$$


Now we correct the four $\lambda_{i}^{3}$ functions to get four new basis functions with vanishing edge and face moments.

$$
\begin{aligned}
& b_{9}=-20 \lambda_{1}^{3}+24 \lambda_{1}^{2}-6 \lambda_{1}, \quad b_{10}=-20 \lambda_{2}^{3}+24 \lambda_{2}^{2}-6 \lambda_{2}, \\
& b_{11}=-20 \lambda_{3}^{3}+24 \lambda_{3}^{2}-6 \lambda_{3}, \quad b_{12}=-20 \lambda_{4}^{3}+24 \lambda_{4}^{2}-6 \lambda_{4} .
\end{aligned}
$$

Under the basis, $u=\sum_{i=1}^{28} c_{i} b_{i}$. As $\mathbb{E}_{j, k}(u)=\mathbb{F}_{i}(u)=0$, we get

$$
c_{i+12}=0, \quad 1 \leq i \leq 16
$$

Hence $u=\sum_{i=1}^{12} c_{i} b_{i}$. Next we show that the remaining 12 coefficients are also zero. An application of the functionals $r_{j} \mathbb{N}_{j, k}, j=1, \cdots, 4, k=1,2,3$, to $u$, yields the following matrix

$$
A=\left(\begin{array}{cccccccccccc}
\frac{1}{30} & \frac{1}{60} & \frac{-1}{60} & \frac{1}{60} & 0 & 0 & 0 & 0 & 2 & 0 & 0 & 0 \\
\frac{1}{60} & \frac{1}{30} & 0 & 0 & \frac{-1}{60} & \frac{1}{60} & 0 & 0 & 2 & 0 & 0 & 0 \\
\frac{1}{30} & \frac{1}{30} & 0 & 0 & 0 & 0 & \frac{-1}{60} & \frac{-1}{60} & 2 & 0 & 0 & 0 \\
\frac{-1}{60} & \frac{1}{60} & \frac{1}{30} & \frac{1}{60} & 0 & 0 & 0 & 0 & 0 & 2 & 0 & 0 \\
0 & 0 & \frac{1}{30} & \frac{1}{30} & \frac{-1}{60} & \frac{-1}{60} & 0 & 0 & 0 & 2 & 0 & 0 \\
0 & 0 & \frac{1}{60} & \frac{1}{30} & 0 & 0 & \frac{-1}{60} & \frac{1}{60} & 0 & 2 & 0 & 0 \\
\frac{1}{60} & \frac{-1}{60} & 0 & 0 & \frac{1}{30} & \frac{1}{60} & 0 & 0 & 0 & 0 & 2 & 0 \\
0 & 0 & \frac{-1}{60} & \frac{-1}{60} & \frac{1}{30} & \frac{1}{30} & 0 & 0 & 0 & 0 & 2 & 0 \\
0 & 0 & 0 & 0 & \frac{1}{60} & \frac{1}{30} & \frac{1}{60} & \frac{-1}{60} & 0 & 0 & 2 & 0 \\
\frac{-1}{60} & \frac{-1}{60} & 0 & 0 & 0 & 0 & \frac{1}{30} & \frac{1}{30} & 0 & 0 & 0 & 2 \\
0 & 0 & \frac{1}{60} & \frac{-1}{60} & 0 & 0 & \frac{1}{30} & \frac{1}{60} & 0 & 0 & 0 & 2 \\
0 & 0 & 0 & 0 & \frac{1}{60} & \frac{-1}{60} & \frac{1}{60} & \frac{1}{30} & 0 & 0 & 0 & 2
\end{array}\right) .
$$

Its determinant is nonzero. Thus, we have $c_{i}=0$ for $i \leq 12$.

It follows from Theorem 2.1 that

Theorem 6.2. With $V_{h, \ell}^{3}=V_{h, H Z 28}$ in (2.1), the finite element solution $u_{h}$ of (2.1) approximates the biharmonic solution $u$ of (1.1) at the second order.

$$
\left|u-u_{h}\right|_{2, h} \leq C h^{2}|u|_{4}
$$

provided that $u \in H^{4}(\Omega) \cap H_{0}^{2}(\Omega)$.

\subsection{The basis functions}

We present a basis for $P_{3}^{+}(K)$ with respect to degrees of freedom defined in (6.1). Let $b_{i}, i=1, \cdots, 8$ and $i=9, \cdots, 12$ be defined in (6.3), (6.4a) and (6.4b), respectively. We first 
give the 12 basis functions for the functionals $\mathbb{N}_{j, k}$ as follows

$$
\begin{aligned}
& \varphi_{1,1}(\mathbf{x})=20 r_{1}\left(b_{1}-b_{3}+b_{5}+2 b_{7}\right)+\frac{r_{1}}{2}\left(b_{10}-b_{11}-b_{12}\right), \\
& \varphi_{1,2}(\mathbf{x})=20 r_{1}\left(b_{2}+b_{3}-b_{5}+2 b_{8}\right)+\frac{r_{1}}{2}\left(-b_{10}+b_{11}-b_{12}\right), \\
& \varphi_{1,3}(\mathbf{x})=-20 r_{1}\left(b_{1}+b_{2}+2 b_{7}+2 b_{8}\right)+\frac{r_{1}}{2}\left(b_{9}+2 b_{12}\right), \\
& \varphi_{2,1}(\mathbf{x})=20 r_{2}\left(-b_{1}+b_{3}+2 b_{5}+b_{7}\right)+\frac{r_{2}}{2}\left(b_{9}-b_{11}-b_{12}\right), \\
& \varphi_{2,2}(\mathbf{x})=-20 r_{2}\left(b_{3}+b_{4}+2 b_{5}+2 b_{6}\right)+\frac{r_{2}}{2}\left(b_{10}+2 b_{11}\right), \\
& \varphi_{2,3}(\mathbf{x})=20 r_{2}\left(b_{1}+b_{4}+2 b_{6}-b_{7}\right)+\frac{r_{2}}{2}\left(-b_{9}-b_{11}+b_{12}\right), \\
& \varphi_{3,1}(\mathbf{x})=20 r_{3}\left(-b_{2}+2 b_{3}+b_{5}+b_{8}\right)+\frac{r_{3}}{2}\left(b_{9}-b_{10}-b_{12}\right), \\
& \varphi_{3,2}(\mathbf{x})=-20 r_{3}\left(2 b_{3}+2 b_{4}+b_{5}+b_{6}\right)+\frac{r_{3}}{2}\left(2 b_{10}+b_{11}\right), \\
& \varphi_{3,3}(\mathbf{x})=20 r_{3}\left(b_{2}+2 b_{4}+b_{6}-b_{8}\right)+\frac{r_{3}}{2}\left(-b_{9}-b_{10}+b_{12}\right), \\
& \varphi_{4,1}(\mathbf{x})=-20 r_{4}\left(2 b_{1}+2 b_{2}+b_{7}+b_{8}\right)+\frac{r_{4}}{2}\left(2 b_{9}+b_{12}\right), \\
& \varphi_{4,2}(\mathbf{x})=20 r_{4}\left(2 b_{1}-b_{4}+b_{6}+b_{7}\right)+\frac{r_{4}}{2}\left(-b_{9}+b_{10}-b_{11}\right), \\
& \varphi_{4,3}(\mathbf{x})=20 r_{4}\left(2 b_{2}+b_{4}-b_{6}+b_{8}\right)+\frac{r_{4}}{2}\left(-b_{9}-b_{10}+b_{11}\right) .
\end{aligned}
$$

These 12 basis functions satisfy

$$
\mathbb{N}_{i, l}\left(\varphi_{j, k}\right)=\delta_{i, j} \delta_{l, k}, \quad i, j=1, \cdots, 4, \quad l, k=1,2,3
$$

and vanish for the remaining functionals in (6.1).

The second four basis functions are for the functionals $\mathbb{F}_{j}$.

$$
\begin{aligned}
& \phi_{1}(\mathbf{x})= 60 \lambda_{1} \lambda_{2} \lambda_{3}+\left(2 \varphi_{1,1}(\mathbf{x})+2 \varphi_{1,2}(\mathbf{x})+\varphi_{1,3}(\mathbf{x})\right) / r_{1} \\
&+\left(2 \varphi_{2,1}(\mathbf{x})+2 \varphi_{2,2}(\mathbf{x})+\varphi_{2,3}(\mathbf{x})\right) / r_{2}+\left(2 \varphi_{3,1}(\mathbf{x})+2 \varphi_{3,2}(\mathbf{x})+\varphi_{3,3}(\mathbf{x})\right) / r_{3} \\
&-2\left(\mathbf{n}_{4}\right)^{T}\left(\left(\frac{\mathbf{n}_{1}}{2 r_{1}}+\frac{\mathbf{n}_{4}}{r_{4}}\right) \varphi_{4,1}(\mathbf{x})+\left(\frac{\mathbf{n}_{2}}{2 r_{2}}+\frac{\mathbf{n}_{4}}{r_{4}}\right) \varphi_{4,2}(\mathbf{x})+\left(\frac{\mathbf{n}_{3}}{2 r_{3}}+\frac{\mathbf{n}_{4}}{r_{4}}\right) \varphi_{4,3}(\mathbf{x})\right), \\
& \phi_{2}(\mathbf{x})=6 \lambda_{1} \lambda_{2} \lambda_{4}+\left(2 \varphi_{1,1}(\mathbf{x})+\varphi_{1,2}(\mathbf{x})+2 \varphi_{1,3}(\mathbf{x})\right) / r_{1} \\
&+\left(2 \varphi_{2,1}(\mathbf{x})+\varphi_{2,2}(\mathbf{x})+2 \varphi_{2,3}(\mathbf{x})\right) / r_{2}+\left(2 \varphi_{4,1}(\mathbf{x})+2 \varphi_{4,2}(\mathbf{x})+\varphi_{4,3}(\mathbf{x})\right) / r_{4} \\
&-2\left(\mathbf{n}_{3}\right)^{T}\left(\left(\frac{\mathbf{n}_{1}}{2 r_{1}}+\frac{\mathbf{n}_{3}}{r_{3}}\right) \varphi_{3,1}(\mathbf{x})+\left(\frac{\mathbf{n}_{2}}{2 r_{2}}+\frac{\mathbf{n}_{3}}{r_{3}}\right) \varphi_{3,2}(\mathbf{x})+\left(\frac{\mathbf{n}_{4}}{2 r_{4}}+\frac{\mathbf{n}_{3}}{r_{3}}\right) \varphi_{3,3}(\mathbf{x})\right), \\
& \phi_{3}(\mathbf{x})=60 \lambda_{1} \lambda_{3} \lambda_{4}+\left(2 \varphi_{1,1}(\mathbf{x})+\varphi_{1,2}(\mathbf{x})+2 \varphi_{1,3}(\mathbf{x})\right) / r_{1} \\
&+\left(2 \varphi_{3,1}(\mathbf{x})+\varphi_{3,2}(\mathbf{x})+2 \varphi_{3,3}(\mathbf{x})\right) / r_{3}+\left(2 \varphi_{4,1}(\mathbf{x})+\varphi_{4,2}(\mathbf{x})+2 \varphi_{4,3}(\mathbf{x})\right) / r_{4} \\
&-2\left(\mathbf{n}_{2}\right)^{T}\left(\left(\frac{\mathbf{n}_{1}}{2 r_{1}}+\frac{\mathbf{n}_{2}}{r_{2}}\right) \varphi_{2,1}(\mathbf{x})+\left(\frac{\mathbf{n}_{3}}{2 r_{3}}+\frac{\mathbf{n}_{2}}{r_{2}}\right) \varphi_{2,2}(\mathbf{x})+\left(\frac{\mathbf{n}_{4}}{2 r_{4}}+\frac{\mathbf{n}_{2}}{r_{2}}\right) \varphi_{2,3}(\mathbf{x})\right), \\
& \phi_{4}(\mathbf{x})=60 \lambda_{2} \lambda_{3} \lambda_{4}+\left(\varphi_{2,1}(\mathbf{x})+2 \varphi_{2,2}(\mathbf{x})+2 \varphi_{2,3}(\mathbf{x})\right) / r_{2} \\
&+\left(\varphi_{3,1}(\mathbf{x})+2 \varphi_{3,2}(\mathbf{x})+2 \varphi_{3,3}(\mathbf{x})\right) / r_{3}+\left(\varphi_{4,1}(\mathbf{x})+2 \varphi_{4,2}(\mathbf{x})+2 \varphi_{4,3}(\mathbf{x})\right) / r_{4} \\
&-2\left(\mathbf{n}_{1}\right)^{T}\left(\left(\frac{\mathbf{n}_{2}}{2 r_{2}}+\frac{\mathbf{n}_{1}}{r_{1}}\right) \varphi_{1,1}(\mathbf{x})+\left(\frac{\mathbf{n}_{3}}{2 r_{3}}+\frac{\mathbf{n}_{1}}{r_{1}}\right) \varphi_{1,2}(\mathbf{x})+\left(\frac{\mathbf{n}_{4}}{2 r_{4}}+\frac{\mathbf{n}_{1}}{r_{1}}\right) \varphi_{1,3}(\mathbf{x})\right) .
\end{aligned}
$$


These 4 basis functions satisfy

$$
\mathbb{F}_{j}\left(\phi_{k}\right)=\delta_{j, k}, \quad j, k=1, \cdots, 4
$$

and vanish for the remaining functionals in (6.1).

At the end we present the 12 basis functions for the functionals $\mathbb{E}_{j, k}$.

$$
\begin{aligned}
& \theta_{1,1}(\mathbf{x})=12 \lambda_{1} \lambda_{2}\left(5 \lambda_{1}-2\right)-2\left(2 \varphi_{1,1}(\mathbf{x})+\varphi_{1,2}(\mathbf{x})+\varphi_{1,3}(\mathbf{x})\right) / r_{1} \\
& +2 \varphi_{2,1}(\mathbf{x}) / r_{2}+2\left(\frac{\mathbf{n}_{1}}{r_{1}}+\frac{\mathbf{n}_{2}}{r_{2}}\right)^{T}\left(\varphi_{3,1}(\mathbf{x}) \mathbf{n}_{3}+\varphi_{4,1}(\mathbf{x}) \mathbf{n}_{4}\right), \\
& \theta_{1,2}(\mathbf{x})=12 \lambda_{1} \lambda_{2}\left(5 \lambda_{2}-2\right)-2\left(2 \varphi_{2,1}(\mathbf{x})+\varphi_{2,2}(\mathbf{x})+\varphi_{2,3}(\mathbf{x})\right) / r_{2} \\
& +2 \varphi_{1,1}(\mathbf{x}) / r_{1}+2\left(\frac{\mathbf{n}_{1}}{r_{1}}+\frac{\mathbf{n}_{2}}{r_{2}}\right)^{T}\left(\varphi_{3,2}(\mathbf{x}) \mathbf{n}_{3}+\varphi_{4,2}(\mathbf{x}) \mathbf{n}_{4}\right), \\
& \theta_{2,1}(\mathbf{x})=12 \lambda_{1} \lambda_{3}\left(5 \lambda_{1}-2\right)-2\left(\varphi_{1,1}(\mathbf{x})+2 \varphi_{1,2}(\mathbf{x})+\varphi_{1,3}(\mathbf{x})\right) / r_{1} \\
& +2 \varphi_{3,1}(\mathbf{x}) / r_{3}+2\left(\frac{\mathbf{n}_{1}}{r_{1}}+\frac{\mathbf{n}_{3}}{r_{3}}\right)^{T}\left(\varphi_{2,1}(\mathbf{x}) \mathbf{n}_{2}+\varphi_{4,1}(\mathbf{x}) \mathbf{n}_{4}\right), \\
& \theta_{2,2}(\mathbf{x})=12 \lambda_{1} \lambda_{3}\left(5 \lambda_{3}-2\right)-2\left(2 \varphi_{3,1}(\mathbf{x})+\varphi_{3,2}(\mathbf{x})+\varphi_{3,3}(\mathbf{x})\right) / r_{3} \\
& +2 \varphi_{1,2}(\mathbf{x}) / r_{1}+2\left(\frac{\mathbf{n}_{1}}{r_{1}}+\frac{\mathbf{n}_{3}}{r_{3}}\right)^{T}\left(\varphi_{2,2}(\mathbf{x}) \mathbf{n}_{2}+\varphi_{4,3}(\mathbf{x}) \mathbf{n}_{4}\right), \\
& \theta_{3,1}(\mathbf{x})=12 \lambda_{1} \lambda_{4}\left(5 \lambda_{1}-2\right)-2\left(\varphi_{1,1}(\mathbf{x})+\varphi_{1,2}(\mathbf{x})+2 \varphi_{1,3}(\mathbf{x})\right) / r_{1} \\
& +2 \varphi_{4,1}(\mathbf{x}) / r_{4}+2\left(\frac{\mathbf{n}_{1}}{r_{1}}+\frac{\mathbf{n}_{4}}{r_{4}}\right)^{T}\left(\varphi_{2,1}(\mathbf{x}) \mathbf{n}_{2}+\varphi_{3,1}(\mathbf{x}) \mathbf{n}_{3}\right), \\
& \theta_{3,2}(\mathbf{x})=12 \lambda_{1} \lambda_{4}\left(5 \lambda_{4}-2\right)-2\left(2 \varphi_{4,1}(\mathbf{x})+\varphi_{4,2}(\mathbf{x})+\varphi_{4,3}(\mathbf{x})\right) / r_{4} \\
& +2 \varphi_{1,3}(\mathbf{x}) / r_{1}+2\left(\frac{\mathbf{n}_{1}}{r_{1}}+\frac{\mathbf{n}_{4}}{r_{4}}\right)^{T}\left(\varphi_{2,3}(\mathbf{x}) \mathbf{n}_{2}+\varphi_{3,3}(\mathbf{x}) \mathbf{n}_{3}\right), \\
& \theta_{4,1}(\mathbf{x})=12 \lambda_{2} \lambda_{3}\left(5 \lambda_{2}-2\right)-2\left(\varphi_{2,1}(\mathbf{x})+2 \varphi_{2,2}(\mathbf{x})+\varphi_{2,3}(\mathbf{x})\right) / r_{2} \\
& 2 \varphi_{3,2}(\mathbf{x}) / r_{3}+2\left(\frac{\mathbf{n}_{2}}{r_{2}}+\frac{\mathbf{n}_{3}}{r_{3}}\right)^{T}\left(\varphi_{1,1}(\mathbf{x}) \mathbf{n}_{1}+\varphi_{4,2}(\mathbf{x}) \mathbf{n}_{4}\right), \\
& \theta_{4,2}(\mathbf{x})=12 \lambda_{2} \lambda_{3}\left(5 \lambda_{3}-2\right)-2\left(\varphi_{3,1}(\mathbf{x})+2 \varphi_{3,2}(\mathbf{x})+\varphi_{3,3}(\mathbf{x})\right) / r_{3} \\
& +2 \varphi_{2,2}(\mathbf{x}) / r_{2}+2\left(\frac{\mathbf{n}_{2}}{r_{2}}+\frac{\mathbf{n}_{3}}{r_{3}}\right)^{T}\left(\varphi_{1,2}(\mathbf{x}) \mathbf{n}_{1}+\varphi_{4,3}(\mathbf{x}) \mathbf{n}_{4}\right), \\
& \theta_{5,1}(\mathbf{x})=12 \lambda_{2} \lambda_{4}\left(5 \lambda_{2}-2\right)-2\left(\varphi_{2,1}(\mathbf{x})+\varphi_{2,2}(\mathbf{x})+2 \varphi_{2,3}(\mathbf{x})\right) / r_{2} \\
& +2 \varphi_{4,2}(\mathbf{x}) / r_{4}+2\left(\frac{\mathbf{n}_{2}}{r_{2}}+\frac{\mathbf{n}_{2}}{r_{4}}\right)^{T}\left(\varphi_{1,1}(\mathbf{x}) \mathbf{n}_{1}+\varphi_{3,2}(\mathbf{x}) \mathbf{n}_{3}\right), \\
& \theta_{5,2}(\mathbf{x})=12 \lambda_{2} \lambda_{4}\left(5 \lambda_{4}-2\right)-2\left(\varphi_{4,1}(\mathbf{x})+2 \varphi_{4,2}(\mathbf{x})+\varphi_{4,3}(\mathbf{x})\right) / r_{4} \\
& 2 \varphi_{2,3}(\mathbf{x}) / r_{2}+2\left(\frac{\mathbf{n}_{2}}{r_{2}}+\frac{\mathbf{n}_{2}}{r_{4}}\right)^{T}\left(\varphi_{1,3}(\mathbf{x}) \mathbf{n}_{1}+\varphi_{3,3}(\mathbf{x}) \mathbf{n}_{3}\right), \\
& \theta_{6,1}(\mathbf{x})=12 \lambda_{3} \lambda_{4}\left(5 \lambda_{3}-2\right)-2\left(\varphi_{3,1}(\mathbf{x})+\varphi_{3,2}(\mathbf{x})+2 \varphi_{3,3}(\mathbf{x})\right) / r_{3} \\
& +2 \varphi_{4,3}(\mathbf{x}) / r_{4}+2\left(\frac{\mathbf{n}_{3}}{r_{3}}+\frac{\mathbf{n}_{4}}{r_{4}}\right)^{T}\left(\varphi_{1,2}(\mathbf{x}) \mathbf{n}_{1}+\varphi_{2,2}(\mathbf{x}) \mathbf{n}_{2}\right), \\
& \theta_{6,2}(\mathbf{x})=12 \lambda_{3} \lambda_{4}\left(5 \lambda_{4}-2\right)-2\left(\varphi_{4,1}(\mathbf{x})+\varphi_{4,2}(\mathbf{x})+2 \varphi_{4,3}(\mathbf{x})\right) / r_{4} \\
& +2 \varphi_{3,3}(\mathbf{x}) / r_{3}+2\left(\frac{\mathbf{n}_{3}}{r_{3}}+\frac{\mathbf{n}_{4}}{r_{4}}\right)^{T}\left(\varphi_{1,3}(\mathbf{x}) \mathbf{n}_{1}+\varphi_{2,3}(\mathbf{x}) \mathbf{n}_{2}\right) .
\end{aligned}
$$


These 12 basis functions satisfy

$$
\mathbb{E}_{i, l}\left(\theta_{j, k}\right)=\delta_{i, j} \delta_{l, k}, \quad 1 \leq i, j \leq 6, \quad l, k=1,2
$$

vanish for the remaining functionals in (6.1).

\section{Numerical Tests}

\subsection{Numerical results in $2 \mathrm{D}$}

We first compute the $2 \mathrm{D}$ biharmonic solution of (1.1), where the exact solution is

$$
u=2^{6} x_{1}^{2}\left(1-x_{1}\right)^{2} x_{2}^{2}\left(1-x_{2}\right)^{2},
$$

on the unit square domain $(0,1)^{2}$. We cut the domain by a north-east line to define the level one grid for the computation. Then each triangle is refined into four congruent triangles, to define the higher level grid.

In the first test, we use the $P_{3}+2 B_{4}$ element, $V_{h, H Z 12}$ in (3.2). As expected, the finite element solution does converge to the exact solution at the second order, shown in Table 7.1.

Table 7.1: The error $e_{h}=u-u_{h}$ and the order of convergence, for (7.1), by the $P_{3}+2 B_{4}$ element.

\begin{tabular}{|c|rc|rc|rc|}
\hline $\mathcal{T}_{k}$ & $\left\|e_{h}\right\|_{0}$ & $h^{n}$ & $\left|e_{h}\right|_{1, h}$ & $h^{n}$ & $\left|e_{h}\right|_{2, h}$ & $h^{n}$ \\
\hline 1 & 0.0774343 & 0.0 & 0.414843 & 0.0 & 4.05172 & 0.0 \\
2 & 0.0322262 & 1.3 & 0.198602 & 1.1 & 2.22543 & 0.9 \\
3 & 0.0018858 & 4.1 & 0.020767 & 3.3 & 0.60045 & 1.9 \\
4 & 0.0000831 & 4.5 & 0.002312 & 3.2 & 0.14839 & 2.0 \\
5 & 0.0000039 & 4.4 & 0.000285 & 3.0 & 0.03598 & 2.0 \\
6 & 0.0000003 & 4.0 & 0.000036 & 3.0 & 0.00882 & 2.0 \\
7 & 0.0000000 & 3.9 & 0.000005 & 3.0 & 0.00219 & 2.0 \\
\hline
\end{tabular}

In the second test, we compute the exact solution (7.1) by the $P_{4}$ non-conforming finite element $V_{h, H Z 15}$ defined in (4.2). As shown by the theorem, the finite element solution converges at the second order in the discrete $H^{2}$ norm, shown in Table 7.2. Comparing with the 12 dof $P_{3}+2 B_{4}$ element, this element is slightly more accurate though it is also of the second order.

Table 7.2: The error $e_{h}=u-u_{h}$ and the order of convergence, for (7.1), by the $P_{4}$ element.

\begin{tabular}{|c|rc|rc|rc|}
\hline $\mathcal{T}_{k}$ & $\left\|e_{h}\right\|_{0}$ & $h^{n}$ & $\left|e_{h}\right|_{1, h}$ & $h^{n}$ & $\left|e_{h}\right|_{2, h}$ & $h^{n}$ \\
\hline 1 & 0.049596736 & 0.0 & 0.270374 & 0.0 & 2.50814 & 0.0 \\
2 & 0.004671002 & 3.4 & 0.045237 & 2.6 & 0.79945 & 1.6 \\
3 & 0.000890065 & 2.4 & 0.010920 & 2.1 & 0.30883 & 1.4 \\
4 & 0.000076285 & 3.5 & 0.001595 & 2.8 & 0.08515 & 1.9 \\
5 & 0.000005480 & 3.8 & 0.000214 & 2.9 & 0.02220 & 1.9 \\
6 & 0.000000364 & 3.9 & 0.000028 & 3.0 & 0.00565 & 2.0 \\
7 & 0.000000023 & 4.0 & 0.000004 & 3.0 & 0.00142 & 2.0 \\
\hline
\end{tabular}

In the third test, we approximate the exact solution (7.1) by the $16 \operatorname{dof} P_{3}+6 B_{6}$ element constructed in [20]. On each triangle $K$ with the barycentric-coordinates $\lambda_{i}$, the finite element 
Table 7.3: The error $e_{h}=u-u_{h}$ and the order of convergence, for (7.1), by the $P_{3}+6 B_{6}$ element.

\begin{tabular}{|c|rc|rc|rc|}
\hline $\mathcal{T}_{k}$ & $\left\|e_{h}\right\|_{0}$ & $h^{n}$ & $\left|e_{h}\right|_{1, h}$ & $h^{n}$ & $\left|e_{h}\right|_{2, h}$ & $h^{n}$ \\
\hline 1 & 0.0950343 & 0.0 & 0.479167 & 0.0 & 3.64488 & 0.0 \\
2 & 0.0601461 & 0.7 & 0.308401 & 0.6 & 2.76527 & 0.4 \\
3 & 0.0092643 & 2.7 & 0.053634 & 2.5 & 1.06496 & 1.4 \\
4 & 0.0009260 & 3.3 & 0.006878 & 3.0 & 0.33413 & 1.7 \\
5 & 0.0000721 & 3.7 & 0.000812 & 3.1 & 0.09269 & 1.8 \\
6 & 0.0000049 & 3.9 & 0.000098 & 3.1 & 0.02400 & 1.9 \\
7 & 0.0000003 & 4.0 & 0.000012 & 3.0 & 0.00605 & 2.0 \\
\hline
\end{tabular}

Table 7.4: The error $e_{h}=u-u_{h}$ and the order of convergence, for (7.1), by the $P_{3}+3 B_{5}+3 B_{6}$ element.

\begin{tabular}{|c|rc|rc|rc|}
\hline $\mathcal{T}_{k}$ & $\left\|e_{h}\right\|_{0}$ & $h^{n}$ & $\left|e_{h}\right|_{1, h}$ & $h^{n}$ & $\left|e_{h}\right|_{2, h}$ & $h^{n}$ \\
\hline 1 & 0.0925868 & 0.0 & 0.473937 & 0.0 & 3.56790 & 0.0 \\
2 & 0.0590571 & 0.6 & 0.304468 & 0.6 & 2.73905 & 0.4 \\
3 & 0.0091445 & 2.7 & 0.053343 & 2.5 & 1.06181 & 1.4 \\
4 & 0.0009744 & 3.2 & 0.007110 & 2.9 & 0.34331 & 1.6 \\
5 & 0.0000806 & 3.6 & 0.000851 & 3.1 & 0.09788 & 1.8 \\
6 & 0.0000056 & 3.8 & 0.000102 & 3.1 & 0.02579 & 1.9 \\
7 & 0.0000004 & 3.9 & 0.000012 & 3.0 & 0.00655 & 2.0 \\
\hline
\end{tabular}

shape functions space is $W_{16}(K)=P_{3}(K)+B_{6}(K)$ where

$$
\begin{aligned}
B_{6}(K)=\lambda_{1} \lambda_{2} \lambda_{3} \operatorname{span}\{ & \left(1-3 \lambda_{1}-2 \lambda_{2}+3 \lambda_{1} \lambda_{2}\right) \lambda_{2},\left(1-3 \lambda_{1}-2 \lambda_{3}+3 \lambda_{1} \lambda_{3}\right) \lambda_{3}, \\
& \left(1-3 \lambda_{2}-2 \lambda_{1}+3 \lambda_{1} \lambda_{2}\right) \lambda_{1},\left(1-3 \lambda_{2}-2 \lambda_{3}+3 \lambda_{2} \lambda_{3}\right) \lambda_{3}, \\
& \left.\left(1-3 \lambda_{3}-2 \lambda_{1}+3 \lambda_{1} \lambda_{3}\right) \lambda_{1},\left(1-3 \lambda_{3}-2 \lambda_{2}+3 \lambda_{2} \lambda_{3}\right) \lambda_{2}\right\} .
\end{aligned}
$$

The dual basis of the finite element space, i.e., the degrees of freedom of the finite element, is defined by

$$
\begin{array}{ll}
v\left(\mathbf{x}_{i}\right), v_{x}\left(\mathbf{x}_{i}\right), v_{y}\left(\mathbf{x}_{i}\right) & \text { at three vertices of } K, \\
v\left(\mathbf{x}_{c}\right) & \text { at the bary-center of } K, \\
\int_{e_{j}} \partial_{\mathbf{n}} v d s, \int_{e_{j}} s \partial_{\mathbf{n}} v d s & \text { on three edges of } K .
\end{array}
$$

The computational results are listed in Table 7.3. The finite element is truly of 2nd order accuracy. The local dimension of this element is 16 , but the $H^{2}$ error is about 3 times of that of the new 12 dof $P_{3}+2 B_{4}$ element.

In the fourth test, we approximate the exact solution (7.1) by the 16 dof $P_{3}+3 B_{5}+3 B_{6}$ element from [4]. On each triangle $K$ with barycentric-coordinates $\lambda_{i}$, the finite element shape functions space is

$$
C_{16}(K)=P_{3}(K)+\lambda_{1} \lambda_{2} \lambda_{3} \operatorname{span}\left\{\lambda_{1} \lambda_{2}, \lambda_{2} \lambda_{3}, \lambda_{1} \lambda_{3}, \lambda_{1}^{2} \lambda_{2}, \lambda_{2}^{2} \lambda_{3}, \lambda_{1} \lambda_{3}^{2}\right\} .
$$

The dual basis of the finite element space, i.e., the degrees of freedom of the finite element, is 
Table 7.5: The error $e_{h}=u-u_{h}$ and the order of convergence, for (7.1), by the $P_{3}+2 B_{5}$ element.

\begin{tabular}{|c|rc|rc|rc|}
\hline $\mathcal{T}_{k}$ & $\left\|e_{h}\right\|_{0}$ & $h^{n}$ & $\left|e_{h}\right|_{1, h}$ & $h^{n}$ & $\left|e_{h}\right|_{2, h}$ & $h^{n}$ \\
\hline 1 & 0.1017755 & 0.0 & 0.496119 & 0.0 & 3.66373 & 0.0 \\
2 & 0.0602523 & 0.8 & 0.308833 & 0.7 & 2.77412 & 0.4 \\
3 & 0.0096740 & 2.6 & 0.055137 & 2.5 & 1.09411 & 1.3 \\
4 & 0.0010644 & 3.2 & 0.007374 & 2.9 & 0.35943 & 1.6 \\
5 & 0.0000901 & 3.6 & 0.000864 & 3.1 & 0.10364 & 1.8 \\
6 & 0.0000064 & 3.8 & 0.000100 & 3.1 & 0.02749 & 1.9 \\
7 & 0.0000004 & 3.9 & 0.000012 & 3.0 & 0.00701 & 2.0 \\
\hline
\end{tabular}

Table 7.6: The error $e_{h}=u-u_{h}$ and the order of convergence, for (7.2), by the $P_{3}+2 B_{4}$ element.

\begin{tabular}{|c|rc|rc|rc|}
\hline $\mathcal{T}_{k}$ & $\left\|e_{h}\right\|_{0}$ & $h^{n}$ & $\left|e_{h}\right|_{1, h}$ & $h^{n}$ & $\left|e_{h}\right|_{2, h}$ & $h^{n}$ \\
\hline 1 & 0.0257005 & 0.0 & 0.155548 & 0.0 & 1.30794 & 0.0 \\
2 & 0.0118945 & 1.1 & 0.082354 & 0.9 & 1.21970 & 0.1 \\
3 & 0.0007061 & 4.1 & 0.009850 & 3.1 & 0.30520 & 2.0 \\
4 & 0.0000431 & 4.0 & 0.001374 & 2.8 & 0.08565 & 1.8 \\
5 & 0.0000039 & 3.4 & 0.000236 & 2.5 & 0.02793 & 1.6 \\
6 & 0.0000004 & 3.2 & 0.000048 & 2.3 & 0.01093 & 1.4 \\
7 & 0.0000001 & 3.0 & 0.000011 & 2.2 & 0.00486 & 1.2 \\
\hline
\end{tabular}

defined by

$$
\begin{array}{ll}
v\left(\mathbf{x}_{i}\right), v_{x}\left(\mathbf{x}_{i}\right), v_{y}\left(\mathbf{x}_{i}\right) & \text { at three vertices of } K, \\
v\left(\mathbf{x}_{c}\right) & \text { at the bary-center of } K, \\
\int_{e_{j}} \partial_{\mathbf{n}} v d s, \int_{e_{j}} s \partial_{\mathbf{n}} v d s & \text { on three edges of } K .
\end{array}
$$

We compute the solution (7.1) by this $C_{16}$ finite element. The computational results are listed in Table 7.4. The finite element is truly of 2 nd order accuracy. The local dimension of this element is 16 , but the $H^{2}$ error is about 3 times of that of the new 12 dof $P_{3}+2 B_{4}$ element.

In the fifth test, we approximate the exact solution (7.1) by the 12 dof $P_{3}+2 B_{5}$ element constructed in [7]. On each triangle $K$ with barycentric-coordinates $\lambda_{i}$, the finite element shape functions space is

$$
G_{12}(K)=P_{3}(K)+\lambda_{1} \lambda_{2} \lambda_{3} \operatorname{span}\left\{\left(\lambda_{1}-\lambda_{1}^{2}-2 \lambda_{2} \lambda_{3}\right),\left(\lambda_{2}-\lambda_{2}^{2}-2 \lambda_{1} \lambda_{3}\right)\right\} .
$$

The dual basis of the finite element space, i.e., the degrees of freedom of the finite element, is

$$
v\left(\mathbf{x}_{i}\right), v_{x}\left(\mathbf{x}_{i}\right), v_{y}\left(\mathbf{x}_{i}\right) \text {, at three vertices of } K, \text { and } \int_{e_{j}} \partial_{\mathbf{n}} v d s \text { on three edges of } K \text {. }
$$

The computational results are listed in Table 7.5. The finite element is truly of 2nd order accuracy. But its $H^{2}$ error is about 3 times of that of the new $P_{3}+2 B_{4}$ element.

In the next three tests, we consider a singular solution

$$
u=2^{5} x^{5 / 2}(1-x)^{2} y^{2}(1-y)^{2}, \quad\left(x_{1}, x_{2}\right) \in(0,1) \times(0,1) .
$$

This solution is not smooth enough, but in the Sobolev space $H^{3-\epsilon}(\Omega)$. We approximate it by the $P_{3}+2 B_{4}$ element, the $P_{3}+6 B_{6}$ element, and the $P_{3}+2 B_{5}$ element. As expected, the finite 
Table 7.7: The error $e_{h}=u-u_{h}$ and the order of convergence, for (7.2), by the $P_{3}+6 B_{6}$ element.

\begin{tabular}{|c|rc|rc|rc|}
\hline $\mathcal{T}_{k}$ & $\left\|e_{h}\right\|_{0}$ & $h^{n}$ & $\left|e_{h}\right|_{1, h}$ & $h^{n}$ & $\left|e_{h}\right|_{2, h}$ & $h^{n}$ \\
\hline 1 & 0.0402600 & 0.0 & 0.267845 & 0.0 & 5.57420 & 0.0 \\
2 & 0.0218671 & 0.9 & 0.147111 & 0.9 & 3.31137 & 0.8 \\
3 & 0.0031348 & 2.8 & 0.023770 & 2.6 & 1.01281 & 1.7 \\
4 & 0.0003147 & 3.3 & 0.003168 & 2.9 & 0.29331 & 1.8 \\
5 & 0.0000253 & 3.6 & 0.000403 & 3.0 & 0.08106 & 1.9 \\
6 & 0.0000018 & 3.8 & 0.000058 & 2.8 & 0.02393 & 1.8 \\
7 & 0.0000001 & 3.8 & 0.000010 & 2.5 & 0.00829 & 1.5 \\
\hline
\end{tabular}

Table 7.8: The error $e_{h}=u-u_{h}$ and the order of convergence, for (7.2), by the $P_{3}+2 B_{5}$ element.

\begin{tabular}{|c|rc|rc|rc|}
\hline $\mathcal{T}_{k}$ & $\left\|e_{h}\right\|_{0}$ & $h^{n}$ & $\left|e_{h}\right|_{1, h}$ & $h^{n}$ & $\left|e_{h}\right|_{2, h}$ & $h^{n}$ \\
\hline 1 & 0.0416108 & 0.0 & 0.167121 & 0.0 & 1.55539 & 0.0 \\
2 & 0.0215182 & 1.0 & 0.133563 & 0.3 & 1.77014 & 0.0 \\
3 & 0.0031883 & 2.8 & 0.021423 & 2.6 & 0.50960 & 1.8 \\
4 & 0.0003534 & 3.2 & 0.002805 & 2.9 & 0.14026 & 1.9 \\
5 & 0.0000309 & 3.5 & 0.000324 & 3.1 & 0.03780 & 1.9 \\
6 & 0.0000023 & 3.8 & 0.000043 & 2.9 & 0.01138 & 1.7 \\
7 & 0.0000002 & 3.8 & 0.000008 & 2.4 & 0.00424 & 1.4 \\
\hline
\end{tabular}

element solution converges at the possible highest order, in all norms, shown in Tables 7.6-7.8. For this problem, the error of the $P_{3}+2 B_{4}$ element is about the same as that of the $P_{3}+2 B_{5}$ element, much smaller than that of the $P_{3}+6 B_{6}$ element.

\subsection{Numerical results in $3 \mathrm{D}$}

We compute the 3D biharmonic solution of (1.1), where the exact solution is

$$
u=2^{10} x_{1}^{2}\left(1-x_{1}\right)^{2} x_{2}^{2}\left(1-x_{2}\right)^{2} x_{3}^{2}\left(1-x_{3}\right)^{2},
$$

on the unit square domain $(0,1)^{3}$.

In the computation, the level one grid is the one shown in Fig. 7.1, cutting the unit cube into 6 tetrahedra. Each grid is refined into a half-sized grid uniformly, to get a higher level grid, shown in Fig. 7.1. In Table 7.9, the error and the convergence order in various norms are listed for the true solution (7.3), by the $P_{4}$ enriched $P_{3}$ nonconforming finite element (6.2). The optimal order of convergence is achieved in Table 7.9, in all norms, confirming the theorem.
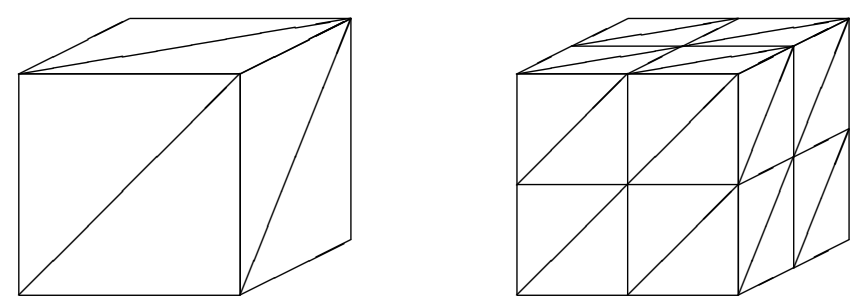

Fig. 7.1. The initial grid for (7.1), and its level 2 refinement. 
Table 7.9: The error $e_{h}=u-u_{h}$ and the order of convergence, for (7.3), by the $P_{3}+8 B_{4}$ element.

\begin{tabular}{|c|rc|rc|rc|}
\hline $\mathcal{T}_{k}$ & $\left\|e_{h}\right\|_{0}$ & $h^{n}$ & $\left|e_{h}\right|_{1, h}$ & $h^{n}$ & $\left|e_{h}\right|_{2, h}$ & $h^{n}$ \\
\hline 1 & 0.0463092 & 0.0 & 0.3918927 & 0.0 & 3.9317928 & 0.0 \\
2 & 0.0203641 & 1.2 & 0.1970587 & 1.0 & 4.5723947 & 0.0 \\
3 & 0.0015314 & 3.7 & 0.0267813 & 2.9 & 1.3978501 & 1.7 \\
4 & 0.0000774 & 4.3 & 0.0033902 & 3.0 & 0.3961007 & 1.8 \\
5 & 0.0000036 & 4.4 & 0.0004229 & 3.0 & 0.1030880 & 1.9 \\
\hline
\end{tabular}

Acknowledgements. The first author was supported by the NSFC Projects 11625101, 91430213 and 11421101.

\section{References}

[1] P. Alfeld and M. Sirvent, The structure of multivariate superspline spaces of high degree, Math. Comp., 57 (1991), 299-308.

[2] J.H. Argyris, I. Fried and D.W. Scharpf, The TUBA family of plate elements for the matrix displacement method, The Aeronautical Journal of the Royal Aeronautical Society, 72 (1968), 514-517.

[3] S.C. Brenner and L.R. Scott, The mathematical theory of finite element methods. Third edition. Texts in Applied Mathematics, 15. Springer, New York, 2008.

[4] H.R. Chen and S.C. Chen, C0-nonconforming elements for a fourth-order elliptic problem, (Chinese) Math. Numer. Sin., 35 (2013), 21-30.

[5] H.R. Chen, S.C. Chen and Z.H. Qiao, C0-nonconforming tetrahedral and cuboid elements for the three-dimensional fourth order elliptic problem, Numer. Math., 24 (2013), 99-119.

[6] P.G. Ciarlet, The Finite Element Method for Elliptic Problems, North-Holland, Amsterdam, 1978.

[7] B. Gao, S. Zhang and M. Wang, A note on the nonconforming finite elements for elliptic problems, J. Comput. Math., 29 (2011), 215-226.

[8] T. Gudi, A new error analysis for discontinuous finite element methods for linear elliptic problems, Math. Comp., 79 (2010), 2169-189.

[9] J. Hu, Y. Huang and S. Zhang, The lowest order differentiable finite element on rectangular grids, SIAM J. Numer. Anal., 49 (2011), 135-1368.

[10] J. Hu, R. Ma and Z. Shi, A new a priori error estimate of nonconforming finite element methods, Sci. China Math., 57 (2014), 887-902.

[11] J. Hu and S. Zhang, The minimal conforming $H^{k}$ finite element spaces on $R^{n}$ rectangular grids, Math. Comp., 84 (2015), 563-579.

[12] J. Hu and S. Zhang, A canonical construction of $H^{m}$-nonconforming triangular elements, Ann. of Appl. Math., 33 (2017), 266-288.

[13] S. P. Mao and Z. C. Shi, On the error bounds of nonconforming finite elements, Sci. China Math., 53 (2010), 2917-2926.

[14] M.J.D. Powell and M. A. Sabin, Piecewise quadratic approximations on triangles, ACM Transactions on Mathematical Software, 3-4 (1977), 316-325.

[15] L.R. Scott and S. Zhang, Finite element interpolation of nonsmooth functions satisfying boundary conditions, Math. Comp., 54 (1990), 483-493.

[16] Z. Shi and M. Wang, Finite Element Methods (Chinese), Science Press, Beijing (2013).

[17] R. Verfürth, A Review of A Posteriori Error Estimation and Adaptive Mesh-Refinement Techniques, Wiley and Teubner, 1996. 
[18] M. Wang and J. Xu, The Morley element for fourth order elliptic equations in any dimensions, Numer. Math., 103 (2006), 155-169.

[19] M. Wang and J. Xu, Minimal finite element spaces for $2 \mathrm{~m}$-th-order partial differential equations in $R^{n}$, Math. Comp., 82 (2013), 25-43.

[20] M. Wang, P. Zu and S. Zhang, High accuracy nonconforming finite elements for fourth order problems, Sci. China Math., 55 (2012), 2183-2192.

[21] A. Ženišek, Alexander Polynomial approximation on tetrahedrons in the finite element method, J. Approx. Theory, 7 (1973), 334-351.

[22] S. Zhang, A family of 3D continuously differentiable finite elements on tetrahedral grids, Appl. Numer. Math., 59 (2009), 219-233.

[23] S. Zhang, A family of differentiable finite elements on simplicial grids in four space dimensions, Math. Numer. Sin., 38 (2016), 309-324. 